\title{
Development of an Integrated Control Strategy Consisting of an Advanced Torque Vectoring Controller and a Genetic Fuzzy Active Steering Controller
}

\author{
Kiumars Jalali \\ Univ. of Waterloo \\ Thomas Uchida \\ Stanford Univ. \\ John McPhee and Steve Lambert \\ Univ. of Waterloo
}

\begin{abstract}
The optimum driving dynamics can be achieved only when the tire forces on all four wheels and in all three coordinate directions are monitored and controlled precisely. This advanced level of control is possible only when a vehicle is equipped with several active chassis control systems that are networked together in an integrated fashion. To investigate such capabilities, an electric vehicle model has been developed with four direct-drive in-wheel motors and an active steering system. Using this vehicle model, an advanced slip control system, an advanced torque vectoring controller, and a genetic fuzzy active steering controller have been developed previously. This paper investigates whether the integration of these stability control systems enhances the performance of the vehicle in terms of handling, stability, path-following, and longitudinal dynamics. An integrated approach is introduced that distributes the required control effort between the inwheel motors and the active steering system. Several test maneuvers are simulated to demonstrate the performance and effectiveness of the integrated control approach, and the results are compared to those obtained using each controller individually. Finally, the integrated controller is implemented in a hardware- and operator-in-the-loop driving simulator to further evaluate its effectiveness.
\end{abstract}

CITATION: Jalali, K., Uchida, T., McPhee, J. and Lambert, S., "Development of an Integrated Control Strategy Consisting of an Advanced Torque Vectoring Controller and a Genetic Fuzzy Active Steering Controller," SAE Int. J. Passeng. Cars Electron. Electr. Syst. 6(1):2013, doi:10.4271/2013-01-0681.

\section{INTRODUCTION}

Over the last two decades, advances in electronics have revolutionized many aspects of automobiles, especially in the areas of engine management and vehicle dynamics safety systems such as the anti-lock braking system (ABS), traction control system (TCS), and electronic stability control (ESC) system. In these cases, the signals generated by the brake or accelerator pedal are modulated by an electronic control unit in order to control the tire slip of individual wheels in severe braking (ABS) or acceleration (TCS) situations, or to control the vehicle yaw rate through individual wheel braking (ESC). It is important to note that the U.S. National Highway Traffic Safety Administration (NHTSA) has passed federal legislation making the installation of ESC mandatory on all passenger cars, multipurpose passenger vehicles, trucks, and buses [1]. The move to improve the safety, comfort, and performance of vehicles has led to an increase in the use of electronic control systems and the introduction of drive-bywire systems. Today, the value added to the modern vehicle by electronic systems is approximately 20 percent. In luxury vehicles, for example, more than 90 control systems are used to control a variety of actuators. It is expected that this rate will consistently increase, reaching over 40 percent by 2015 [2].

Integrating various electronic control systems offers the potential to optimize driving behavior independently of the driving maneuver through the individual control and allocation of traction, steering, and braking forces. These unique features create new opportunities for controlling the 
driving dynamics of a vehicle in ways that were not possible in the past. For example, integrating the active braking and active steering systems can avoid the vehicle side-pushing behavior when braking on a $\mu$-split road []] . In addition, by integrating all chassis control systems, sensor signals can be broadcast across the entire network of controllers, thereby avoiding sensor redundancy and reducing costs.

Recently, electric vehicles (EVs) have attracted a great deal of interest as an elegant solution to environmental and energy problems. EVs have no tailpipe emissions because they have no fuel, combustion, or exhaust systems, and they have achieved driving performance and efficiency metrics that are comparable to those of conventional internal combustion engine vehicles. Critics proclaim that EVs are simply "elsewhere emission vehicles" because they transfer emissions from the tailpipe to the smokestack. However, in the ideal scenario, EVs would be charged using renewable energy sources such as hydro, solar, or wind.

\section{Advanced Stability Control System through Networked Chassis}

Until the 1980s, chassis technology (which directly determines the dynamic performance of a vehicle) advanced exclusively within the mechanical engineering framework. Following the mid-1980s invention and practical application of the four-wheel-steering (4WS) system, the field of vehicle dynamics performance became a main stream of research and development for control technology. Since then, research and development of vehicle dynamics performance has been carried out as a collaboration between mechanical engineering and control engineering.

Current chassis control systems are distinguished by the way the individual subsystems work. While some subsystems affect only one dynamic domain (i.e., longitudinal, lateral, or vertical dynamics), many subsystems can influence two of the three domains, as illustrated in Figure 1. For instance, a torque vectoring system can influence both the longitudinal and lateral dynamics of a vehicle. Furthermore, the influences of individual subsystems are tightly coupled through the tireroad characteristics, especially during extreme maneuvers. Thus, the optimum driving dynamics can be achieved only when the tire forces on all four wheels and in all three coordinate directions can be influenced and controlled precisely. In order to achieve this level of control, the vehicle must not only be equipped with various active chassis subsystems, but these subsystems must be networked together, cooperatively controlling the tire forces to meet the driving dynamics, safety, and comfort requirements [4]].

Taking advantage of the strengths of each subsystem, the ideal stability control system can be obtained by activating the most effective subsystem or subsystems based on the driving maneuver and road conditions. For example, an ESC system uses braking intervention of individual wheels in order to influence the longitudinal forces on the tires and, ultimately, the yaw behavior of the vehicle. However, in many situations, a vehicle can be stabilized without applying the brakes. For example, if the driver wishes to maintain a constant vehicle speed while negotiating a curve, torque vectoring techniques can be used to control the driving dynamics without slowing the vehicle.

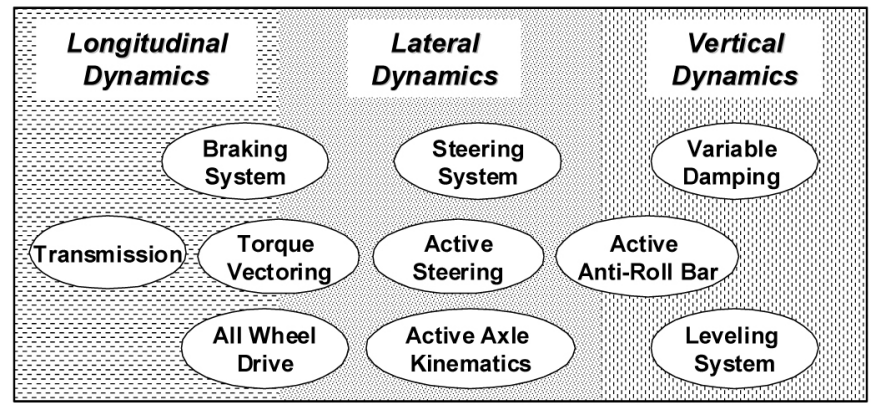
Figure 1. Domains affected by driveline and chassis
subsystems [4]

Figure 2 illustrates the interdependencies among longitudinal, lateral, and vertical dynamics. As indicated, a friction ellipse couples the longitudinal and lateral tire forces, a "longitudinal friction coefficient versus slip ratio" relationship couples the longitudinal and vertical tire forces, and a "lateral friction coefficient versus slip angle" relationship couples the vertical and lateral forces of a tire. Thus, most vehicle control principles can be related to the linear and nonlinear characteristics of the tire-road contacts. The factors influencing the nature of this contact can be summarized as direct effects of the steering angle, slip angle, and camber angle of the tires on lateral forces, and direct effects of traction and braking intervention on longitudinal forces. The wheel vertical load, however, influences both longitudinal and lateral forces directly by defining the maximum possible adhesion potential.

The fundamental question of which configurations are both effective and feasible given a specific set of driving conditions can be answered only if the strengths and limitations of each active chassis subsystem have been identified. A popular method of addressing such issues is to analyze the effects of each subsystem on the resulting tireroad friction ellipse or circle. Since the driver is limited by the friction constraints of the tires, the vehicle controls are expected to provide the driver with predictable authority over longitudinal and lateral accelerations, within the physical constraints of the vehicle "friction circle", and subject to perceived customer acceptability of the frequency- and amplitude-dependence of the vehicle responses [5]. Therefore, given that friction limits change with speed, road surface conditions, and so forth, these vehicle control systems are required to provide adequate feedback of such changes. The illustrations shown in Figure 3, presented by Toyota [ 6 ], indicate the domain of operation of some typical vehicle control systems. Although the diagrams should not be taken too literally, they clearly underline the fact that integrated control can enlarge the dynamic response domain of the 
vehicle by taking advantage of the control system with the most effectiveness for a particular driving maneuver and set of road conditions.

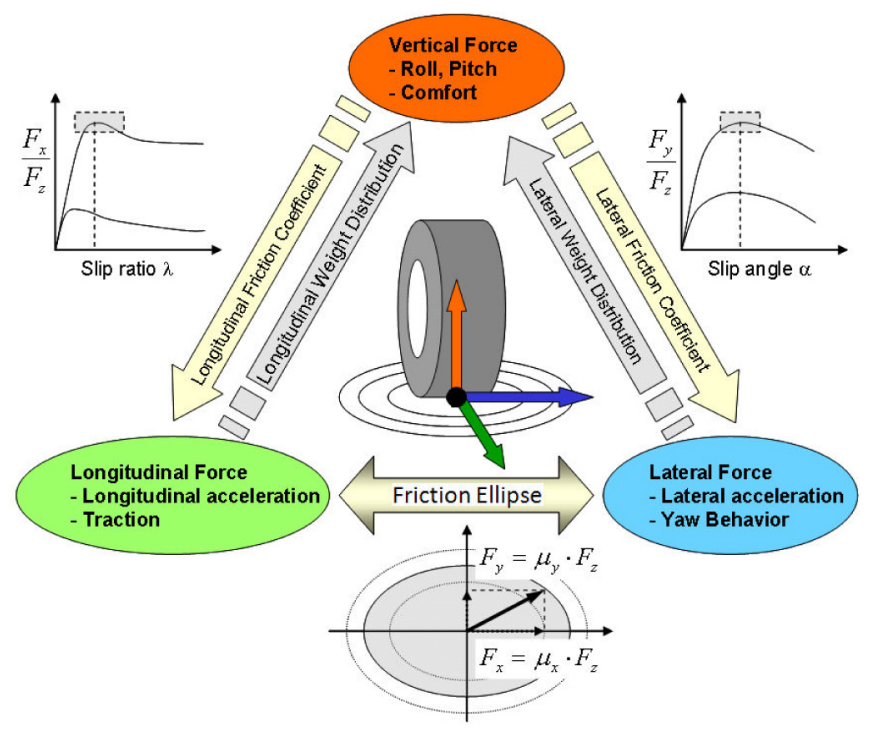

Figure 2. Interdependencies among longitudinal, lateral, and vertical dynamics
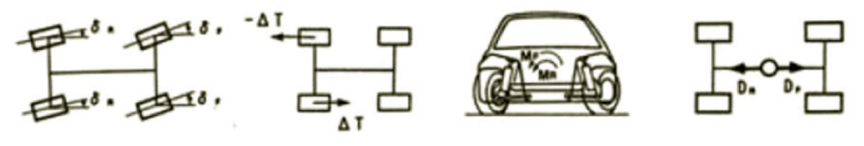

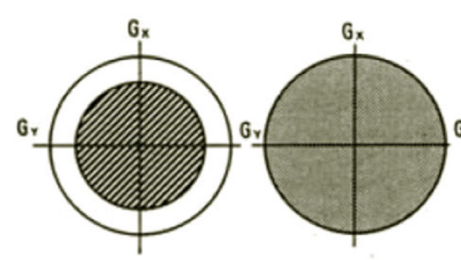

Active Steering
Left-to-Right Torque Vectoring

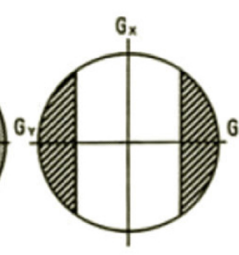

Active Suspension

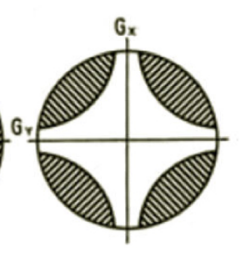

Front-to-Rear Torque Vectoring
Figure 3. Effective range of various control systems based on the resulting tire-road friction circle [6]

The majority of the stability control systems currently on the market stabilize the vehicle in critical driving situations by intervening with only one type of active chassis subsystem at a time, which limits the performance of the vehicle. Recently, there has been a move towards networking the individual subsystems in order to take advantage of synergies and increase the performance of the vehicle. Until very recently, however, mainly due to marketing strategies, chassis subsystems have been treated as stand-alone systems in a socalled "coexistence" architecture, which requires no overhead but still suffers from suboptimal performance. This architecture can lead to a situation in which, for instance, a vehicle with four active chassis subsystems is equipped with as many as four independent sets of sensors, state estimators, reference models, and state controllers $[\underline{3}, \underline{4}]$. Since the simultaneous actuation of these subsystems may affect the same degrees-of-freedom of the vehicle and have counterproductive results, the overlapping of actuator effort must be addressed in a more coordinated way. One solution to this problem is a "hierarchical coexistence" of the subsystems with a unidirectional flow of information, where one system acts independently and the others adapt as necessary.

An "integrated" approach is a more sophisticated means of addressing the coordination of several actuators [ $[\underline{5}, \underline{7}]$. In this approach, each chassis subsystem has one basic function. In contrast to the coexistence approach, there is only one set of sensors, one state estimator, one reference model, and one state controller. Based on the desired and actual behavior of the vehicle, the system can calculate the required generalized forces and moments to maintain the course desired by the driver. These generalized forces and moments are then applied by the actuators based on their effectiveness, ensuring the best overall safety, ride quality, and driving pleasure.

\section{DEVELOPMENT OF AN INTEGRATED CHASSIS CONTROL SYSTEM}

A two-passenger, all-wheel-drive urban electric vehicle (AUTO21EV) with four direct-drive in-wheel motors and an active steering system has been designed and developed at the University of Waterloo. The AUTO21EV is modeled in both ADAMS/View and MapleSim, and is an exciting platform on which to apply advanced motion control techniques. Figure 4 illustrates the AUTO21EV, which has a similar configuration to the commercially-available Smart fortwo.

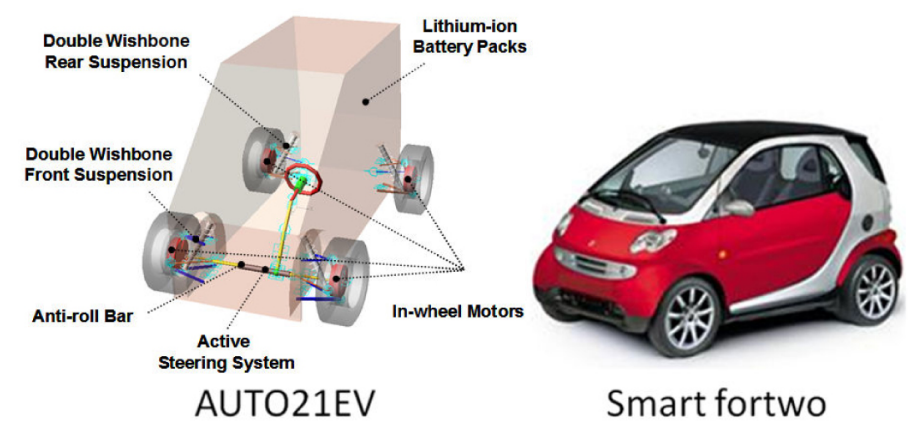

Figure 4. AUTO21EV concept vehicle (left) and Smart fortwo [8] (right)

Table 1 lists some of the relevant parameters used for the AUTO21EV model. Based on this vehicle model, an advanced fuzzy slip control system [9] was developed and tested using some predefined test maneuvers and a novel path-following and speed-controlling driver model [10]. A genetic fuzzy yaw moment controller was also developed [11], the objective of which was to determine the corrective yaw moment required to minimize the vehicle yaw rate and sideslip errors. The genetically-tuned fuzzy yaw moment 
controller acts as a high-level supervisory module that assigns tasks to a lower-level torque vectoring controller [12], which then distributes the task of generating the calculated corrective yaw moment to the in-wheel motors. The developed advanced torque vectoring controller consists of left-to-right and front-to-rear torque vectoring components, which work together to distribute the calculated corrective yaw moment in an integrated approach. Moreover, a genetic fuzzy active steering controller was developed using the AUTO21EV vehicle model [13], and the performance and effectiveness of this controller were studied using a driving simulator. In this work, we investigate whether the integration of these stability control systems enhances the performance of the vehicle in terms of handling, stability, path-following, and longitudinal dynamics. An integrated approach is introduced that distributes the required control effort between the in-wheel motors and the active steering system.

Table 1. AUTO21EV model parameters

\begin{tabular}{|l|c|c|}
\hline Parameter & Value & Unit \\
\hline Vehicle mass $(M)$ & 1075 & $\mathrm{~kg}$ \\
\hline Vehicle yaw moment of inertia $\left(\mathrm{I}_{\mathrm{zz}}\right)$ & 1171 & $\mathrm{~kg} \mathrm{~m}$ \\
\hline Distance from CG to front axle $(\mathrm{a})$ & 0.82 & $\mathrm{~m}$ \\
\hline Distance from CG to rear axle $(\mathrm{b})$ & 0.98 & $\mathrm{~m}$ \\
\hline Front track width $\left(\mathrm{t}_{\mathrm{f}}\right)$ & 1.275 & $\mathrm{~m}$ \\
\hline Rear track width $\left(\mathrm{t}_{\mathrm{r}}\right)$ & 1.35 & $\mathrm{~m}$ \\
\hline Front tire cornering stiffness $\left(\mathrm{C}_{\alpha \mathrm{F}}\right)$ & 45570 & $\mathrm{~N} / \mathrm{rad}$ \\
\hline Rear tire cornering stiffness $\left(\mathrm{C}_{\alpha \mathrm{R}}\right)$ & 45570 & $\mathrm{~N} / \mathrm{rad}$ \\
\hline Tire size & $175 / 55 \mathrm{R} 15$ & - \\
\hline Tire unloaded radius $(\mathrm{r})$ & 0.29 & $\mathrm{~m}$ \\
\hline
\end{tabular}

\section{INTEGRATION OF THE ADVANCED TORQUE VECTORING AND GENETIC FUZZY ACTIVE STEERING CONTROLLERS}

As mentioned above, the optimum driving dynamics can be achieved only when the tire forces on all four wheels and in all three coordinate directions are monitored and controlled precisely. This advanced level of control is possible only when the vehicle is equipped with several active chassis control systems that are networked together in an integrated fashion. Taking advantage of the strengths of each active chassis subsystem, the ideal traction and stability performance of the vehicle can be obtained by activating the subsystem or subsystems that will be most effective given the deviation between the desired and actual behavior of the vehicle. The performance and effectiveness of the advanced torque vectoring controller (ATVC) and the genetic fuzzy active steering controller (GFASC) were studied and evaluated individually in previous papers $[\underline{12}, \underline{13}]$. In this work, we investigate whether the integration of these stability control systems enhances the performance of the vehicle in terms of handling, stability, path-following, and longitudinal dynamics. An integrated approach is introduced that distributes the required control effort between the in-wheel motors and the active steering system.

The GFASC developed in [13] has confirmed that superimposing the steering input provided by the driver with a correction generated by the active steering system is considered to be a continuous process, and is not perceived by the driver as being disruptive. It is also advantageous to employ steering intervention rather than braking or driving individual wheels when controlling the vehicle on slippery surfaces, since steering intervention requires less frictional force between the tire and the road to generate a corrective yaw moment. However, the GFASC is not of substantial help when the vehicle is driven near its handling limits due to its limited range of effectiveness (caused by actuator restrictions). In [12], on the other hand, it has been confirmed that the ATVC is very effective at improving vehicle stability and handling, even when driving the vehicle near its handling limits. It has also been observed, however, that using the inwheel motors to generate a corrective yaw moment can cause oscillations in the vehicle states, which might be perceived by the driver as being annoying. Thus, the GFASC and ATVC appear to be complementary.

In this work, the integration of the ATVC and GFASC is realized by using the activation function illustrated in Figure 5. This activation function is a standard Gaussian curve generated using the following exponential function:

$$
\chi_{\text {ATVC }}\left(\delta_{\text {corr }}\right)=\left\{\begin{array}{cc}
\exp \left[\frac{-\left(\delta_{\text {corr }}-\delta_{\text {corr } \max }\right)^{2}}{2 \sigma^{2}}\right] \cdot 100 \% & ,\left|\delta_{\text {corr }}\right| \leq 3^{\circ} \\
100 \% & ,\left|\delta_{\text {corr }}\right|>3^{\circ}
\end{array}\right.
$$

where $\chi_{A T V C}\left(\delta_{c o r r}\right)$ is the ATVC activation function, which is defined as a function of the corrective steering angle $\left(\delta_{\text {corr }}\right) ; \delta_{\text {corr, } \max }$ is the actuator range limit of the active steering controller, which is set to $3^{\circ}[\underline{13}]$; and $\sigma$ is the standard deviation, which is set to 0.7 in order to form the bell curve shown in Figure 5. Note that the shape of this activation function is designed such that the contribution of the ATVC is introduced gradually rather than abruptly. In other words, depending on the driving conditions and the difference between the desired and actual behavior of the vehicle, the GFASC first attempts to stabilize the vehicle without receiving any support from the ATVC (since the ATVC can have a negative effect on driving enjoyment). As the required corrective steering angle increases, the activation function gradually activates the ATVC to support the GFASC in its effort to stabilize the vehicle. If the required corrective steering angle is larger than $3^{\circ}$, thus exceeding the actuator range limit of the active steering controller, the activation function fully activates the ATVC and both controllers are fully deployed to help stabilize the vehicle. In critical or 
emergency driving situations, it is acceptable to sacrifice some amount of passenger comfort to maintain vehicle stability and, ultimately, passenger safety.

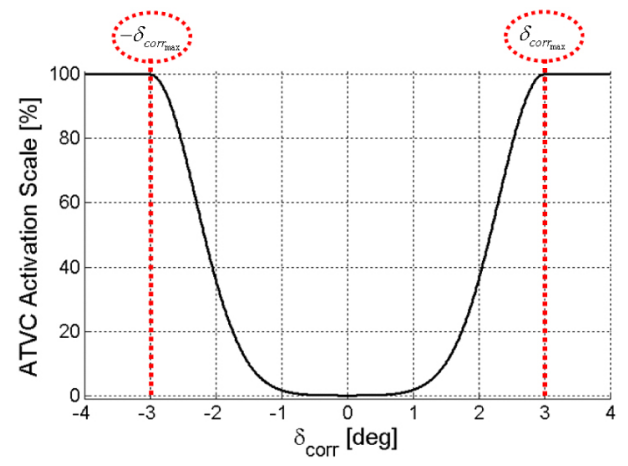

Figure 5. Activation function used for the integration of the ATVC and GFASC

\section{TEST MANEUVERS FOR EVALUATING THE EFFECTIVENESS OF THE INTEGRATED CONTROL STRATEGY}

Many test maneuvers have been developed for evaluating the quality of the handling and performance characteristics of a vehicle. Many of these test maneuvers are based on ideal driving conditions, and some of them are motivated by the examination methods typically used for control systems, such as step-steer and swept-sine-steer maneuvers. An extensive overview of different test maneuvers and their detailed descriptions can be found in the publications of Roenitz, Braess, and Zomotor $[14,15]$. A comprehensive evaluation of the dynamic characteristics of a vehicle and the effectiveness of different chassis control systems can be achieved only when the results obtained from a variety of test maneuvers are combined and evaluated as a whole. Several test maneuvers have been used in the past to provide important information about different aspects of the dynamic behavior of the vehicle and the effectiveness of each individual chassis control system, namely the advanced slip control system [9], the advanced torque vectoring controller [12], and the genetic fuzzy active steering controller [13]. In this work, we use the same test maneuvers to evaluate the effectiveness of our integrated chassis control strategy. These test maneuvers are chosen such that all aspects of vehicle dynamics are addressed and act to quantify the advantages of each control method. A comprehensive description of the chosen test maneuvers and the specific requirements for a desirable vehicle response during each maneuver can be found in [16] In order to evaluate the performance and effectiveness of the integrated control strategy, the AUTO21EV is driven through four test maneuvers.

\section{ISO Double-Lane-Change Maneuver}

The performance of the integrated control system consisting of the ATVC and the GFASC is first evaluated by driving the AUTO21EV through the ISO 3888 double-lanechange maneuver [17] with an initial speed of $75 \mathrm{~km} / \mathrm{h}$ and using the path-following driver model developed previously [10]. Figure 6 illustrates the vehicle trajectory and demonstrates that the driver is able to negotiate the maneuver without striking the cones when the integrated control strategy is used. Note that the path-following driver model is not able to negotiate this maneuver at higher speeds without striking the cones when only the GFASC is active [13].

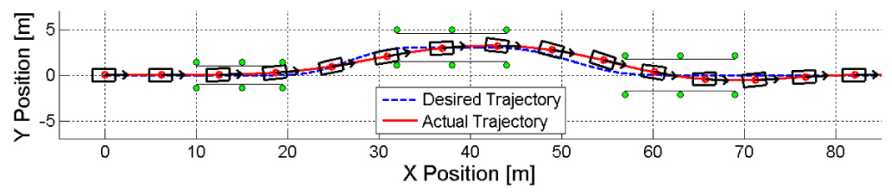

Figure 6. Desired and actual vehicle trajectories when driving through the double-lane-change maneuver with an initial speed of $75 \mathrm{~km} / \mathrm{h}$ using the path-following driver model and the integrated control of the ATVC and GFASC

Figure 7 illustrates the vehicle yaw rate and sideslip angle during this maneuver. In contrast to the individual performance of the ATVC and GFASC, the integrated control approach using both controllers is able to match the actual vehicle yaw rate with the desired yaw rate (which is calculated using a reference bicycle model). Note that the oscillations in the actual vehicle yaw rate that were observed when the ATVC was used on its own [12] are not present when the integrated control approach is used. Figure 8-a illustrates the vehicle lateral acceleration as a function of time. As can be seen, the lateral acceleration when using the integrated control approach is, in some regions, similar to that observed when using the GFASC; in other regions, it is similar to that observed when using the ATVC. At the handling limits, oscillations can be seen in the lateral acceleration of the vehicle, which are caused by the activation of the in-wheel motors, but they are mostly damped out. Figure 8-b confirms that, except during the second lane change, the driver requires about the same amount of steering wheel input as is the case when only the ATVC is used.

Figure 8-c illustrates the vehicle speed during the doublelane-change maneuver, and confirms that the vehicle loses the least amount of speed when the integrated control approach is used. This lack of deceleration can be attributed to the fact that only relatively small steering angles are needed to negotiate the maneuver (Figure 8-b) and the fact that the couples generated at the front and rear axles do not slow the vehicle. As a result, the speed reduction during this maneuver when using the integrated control approach is even smaller than that observed when the genetic fuzzy yaw moment controller (GFYMC) is active [11]. Figure 8-d illustrates the handling performance of the vehicle and 
indicates that the hysteresis of this plot is less than that of the analogous plots for the GFASC and the ATVC. In other words, the responsiveness and agility of the vehicle are considerably improved compared to the cases where only individual controllers (namely, the GFASC and the ATVC) are active. However, the responsiveness and agility of the vehicle are not as good as they are when the ideal (but not directly realizable) GFYMC is used to apply the required corrective yaw moment directly to the vehicle center of gravity.
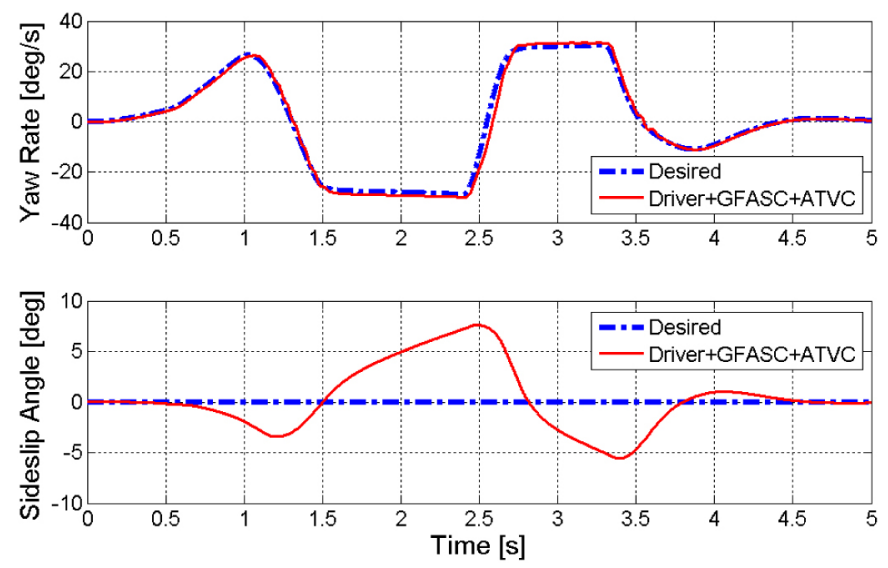

Figure 7. Desired and actual vehicle yaw rates (top) and sideslip angles (bottom) when driving through the double-lane-change maneuver with an initial speed of 75 $\mathrm{km} / \mathrm{h}$ using the driver model and the integrated control of the ATVC and GFASC
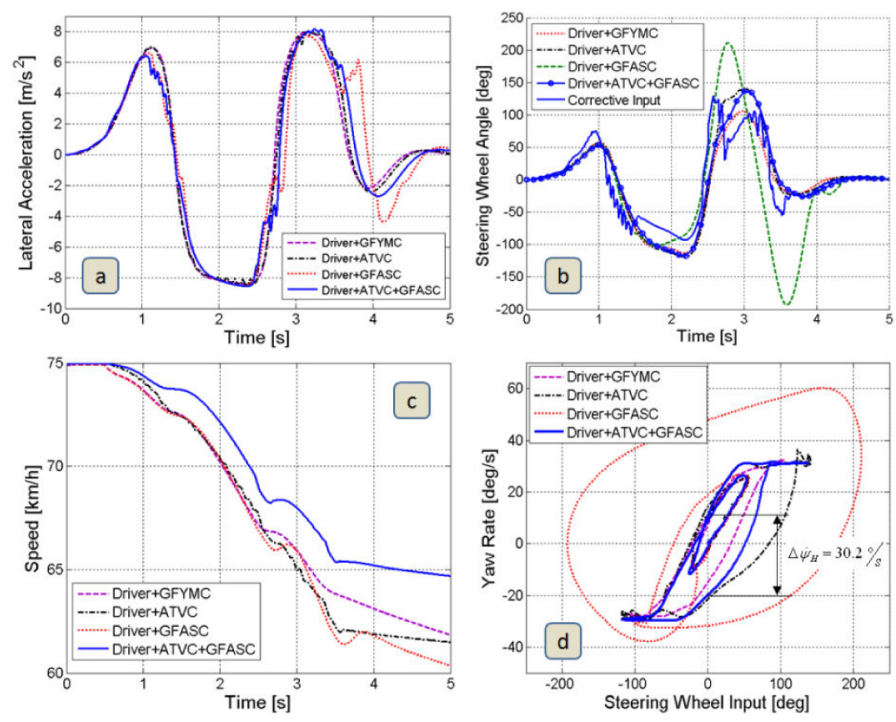

Figure 8. (a) Lateral acceleration, (b) steering wheel angle, and (c) vehicle speed as functions of time; and (d) vehicle yaw rate as a function of the steering wheel input when driving through the double-lane-change maneuver with the GFYMC, the ATVC, the GFASC, and the integrated control of the ATVC and GFASC
Figure 9 illustrates the torque of each in-wheel motor during the double-lane-change maneuver. Comparing this figure to Figure 12 in [12], it is clear that the use of the ATVC has been reduced to a minimum by the activation function. In other words, the ATVC is activated only when the GFASC is unable to stabilize the vehicle on its own, which generally occurs only when the vehicle is driven near its handling limits. Figure 12 indicates that the GFASC is able to stabilize the vehicle most of the time, and the ATVC is only activated at three time periods. For instance, between 2.4 and 2.65 seconds of the simulation, during which time the vehicle experiences the maximum lateral acceleration of 8.5 $\mathrm{m} / \mathrm{s}^{2}$ (Figure $8-\mathrm{a}$ ), the left-to-right torque vectoring controller has ordered the left wheels to brake and the right wheels to accelerate, thereby generating a positive corrective yaw moment to compensate for an oversteering situation, where the actual yaw rate is larger than the desired one (Figure 7).
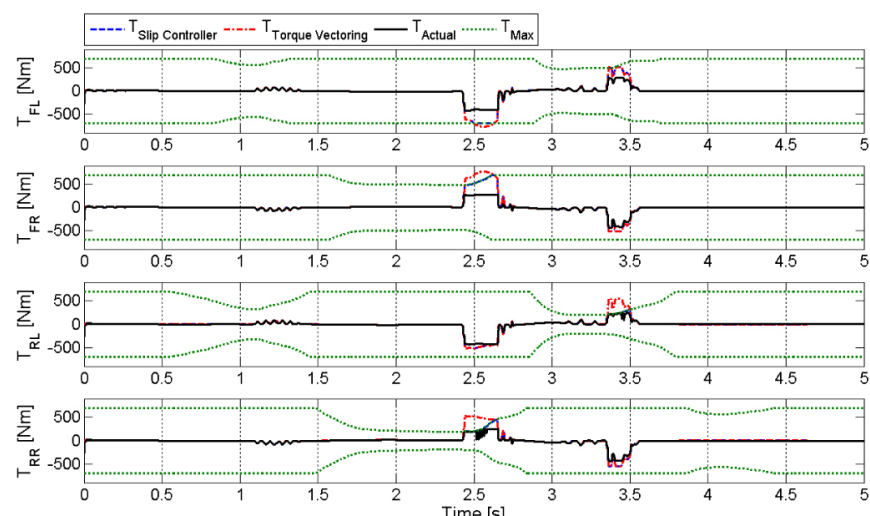

Figure 9. Requested and actual motor torques at each wheel when driving through the double-lane-change maneuver using the driver model with the integrated control of the ATVC and GFASC

From the torque vectoring ratios shown in Figure 10, it can be confirmed that the front-to-rear torque vectoring controller supports both the GFASC and the left-to-right torque vectoring controller in a coordinated effort to stabilize the vehicle. For instance, between 2.4 and 2.65 seconds, the front-to-rear torque vectoring controller has requested that the front motors generate up to $61 \%$ of the total required corrective yaw moment. Note that generating more torque with the front motors and less with the rear ones reduces the lateral force potential at the front axle and increases that at the rear axle. The asymmetric lateral force potentials on the front and rear axles help to generate the required corrective yaw moment. The activation of the front-to-rear torque vectoring controller can also be confirmed in Figure 9. For instance, between 2.4 and 2.65 seconds, the front-to-rear torque vectoring controller has requested the front-left motor to generate up to $770 \mathrm{Nm}$ of braking torque and the frontright motor to generate up to $770 \mathrm{Nm}$ of driving torque while, at the same time, the controller has requested up to $510 \mathrm{Nm}$ of braking and driving torques from the rear-left and rear- 


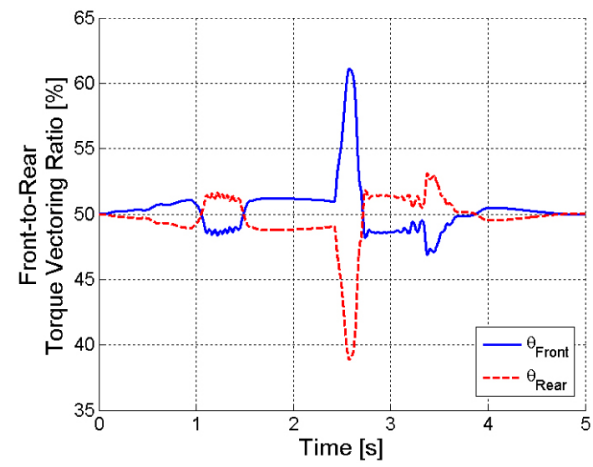

Figure 10. Front-to-rear torque vectoring ratios when driving through the double-lane-change maneuver using the driver model with the integrated control of the ATVC and GFASC

right motors, respectively. Note that, although the controller has requested large motor torques from the front motors, the motors are not powerful enough to generate the requested torques at a speed of $68 \mathrm{~km} / \mathrm{h}$. The effects seen in Figure 9 can also be confirmed by looking at the tire traction potentials and tire slip ratios shown in Figures 11 and $\underline{12}$, respectively. For instance, the maximum traction potential of the rear-left tire is only exceeded once, at 3.35 seconds (Figure 11). From Figures 9 and 12 , it can be confirmed that the requested motor torque at the rear-left wheel is restricted by the slip controller during this time period in order to prevent tire spinout. Thus, the plot of the traction potential of the rear-left tire exceeds the maximum traction potential of 1 because the lateral force of the tire has exceeded its limit. The same explanation is valid for the rear-right tire when its traction potential is exceeded at 2.45 seconds.
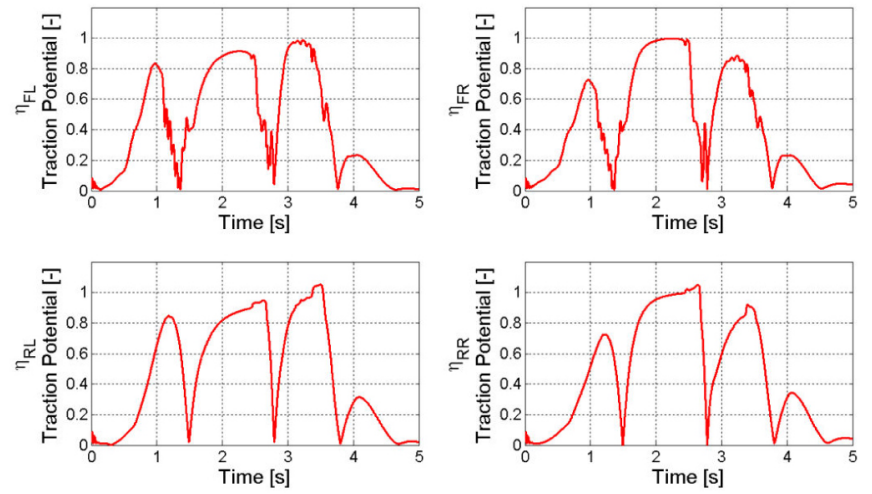

Figure 11. Traction potential of each tire when driving through the double-lane-change maneuver using the driver model with the integrated control of the ATVC and GFASC

Table 2 summarizes the vehicle response during the double-lane-change maneuver when the driver model is used with the integrated control of the ATVC and GFASC. The vehicle response is compared to the cases where the driver model is used with no stability controller, with the GFYMC, with the ATVC, and with the GFASC [16]. Comparing different parameters of the vehicle response during the double-lane-change maneuver, it can be seen that the integrated controller performs better than either of the individual controllers (namely, the ATVC and the GFASC) in terms of all the decisive parameters that describe the handling, stability, and longitudinal dynamics of the vehicle. Moreover, with respect to the handling of the vehicle, the integrated control approach has reduced $|\beta|_{\max }$ and $|\dot{\psi}|_{\max }$ by about the same amount as the ideal (but not directly realizable) GFYMC. The maximum lateral acceleration of the vehicle $\left(\left|a_{y}\right|_{\max }\right)$ is also about the same as it is when the GFYMC is active; however, when the integrated control system is active, the driver requires a larger maximum steering wheel angle to negotiate the maneuver. In addition, the hysteresis of the performance plot $\left(\Delta \dot{\psi}_{H}\right)$ is about 1.4 times larger than that observed when the GFYMC is active. However, the speed lost during the maneuver is less than that lost when the GFYMC is active. Altogether, the integrated control approach is considered to be very effective at improving the handling and stability characteristics of the vehicle. Since the least amount of speed is lost during the maneuver when the integrated control approach is used, it is considered to be the most effective controller for improving the longitudinal dynamics of the vehicle.

Table 2. Vehicle response during the double-lane-change maneuver using the driver model without a controller (AUTO21EV), with the GFYMC, with the ATVC, with the GFASC, and with the integrated control of the ATVC and GFASC [16]

\begin{tabular}{|c|c|c|c|c|c|c|}
\hline Parameter & $|\beta|_{\max }$ & $|\dot{\psi}|_{\max }$ & $\left|a_{y}\right|_{\max }$ & $\left|\delta_{S W}\right|_{\max }$ & $\Delta \dot{\psi}_{H}$ & $v_{\text {lost }}$ \\
\hline AUTO21EV & $26.3^{\circ}$ & $115.4^{\circ} / \mathrm{s}$ & $8.2 \mathrm{~m} / \mathrm{s}^{2}$ & $545^{\circ}$ & $163.8^{\circ} / \mathrm{s}$ & $20.5 \mathrm{~m} / \mathrm{s}$ \\
\hline GFYMC & $6.2^{\circ}$ & $31.2^{\circ} / \mathrm{s}$ & $8.4 \mathrm{~m} / \mathrm{s}^{2}$ & $108^{\circ}$ & $21.0^{\circ} / \mathrm{s}$ & $13.2 \mathrm{~m} / \mathrm{s}$ \\
\hline ATVC & $6.1^{\circ}$ & $36.4^{\circ} / \mathrm{s}$ & $8.4 \mathrm{~m} / \mathrm{s}^{2}$ & $140^{\circ}$ & $35.5^{\circ} / \mathrm{s}$ & $13.5 \mathrm{~m} / \mathrm{s}$ \\
\hline GFASC & $13.6^{\circ}$ & $60.1^{\circ} / \mathrm{s}$ & $8.4 \mathrm{~m} / \mathrm{s}^{2}$ & $211^{\circ}$ & $79.5^{\circ} / \mathrm{s}$ & $14.6 \mathrm{~m} / \mathrm{s}$ \\
\hline ATVC+GFASC & $7.5^{\circ}$ & $31.5^{\circ} / \mathrm{s}$ & $8.5 \mathrm{~m} / \mathrm{s}^{2}$ & $136^{\circ}$ & $30.2^{\circ} / \mathrm{s}$ & $10.3 \mathrm{~m} / \mathrm{s}$ \\
\hline
\end{tabular}




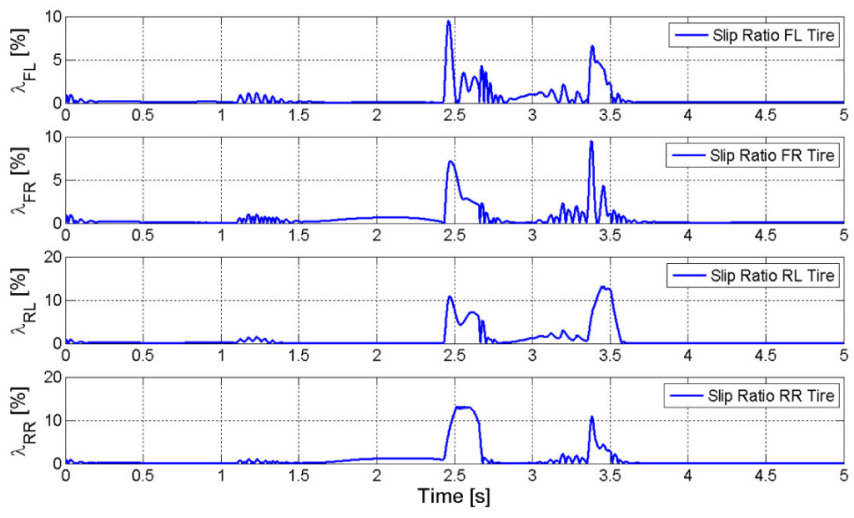

Figure 12. Tire slip ratios when driving through the double-lane-change maneuver using the driver model with the integrated control of the ATVC and GFASC

\section{Step-Steer Response Maneuver}

In order to evaluate the performance of the vehicle using the integrated control of the ATVC and GFASC in a stepsteer response maneuver, the vehicle yaw rate, sideslip angle, and lateral acceleration as functions of time are observed. Figure 13-a illustrates the fixed step-steer input and the equivalent corrective steering input generated by the GFASC. As can be seen, the intervention of the GFASC is almost the same as that observed when the GFASC is used on its own [13]. In other words, the GFASC is able to match the behavior of the vehicle to that of the desired bicycle model without requiring a substantial amount of support from the ATVC. As a result, the vehicle experiences a lateral acceleration of $4.3 \mathrm{~m} / \mathrm{s}^{2}$, which is similar to that obtained when the GFASC is used on its own (Figure 13-b).
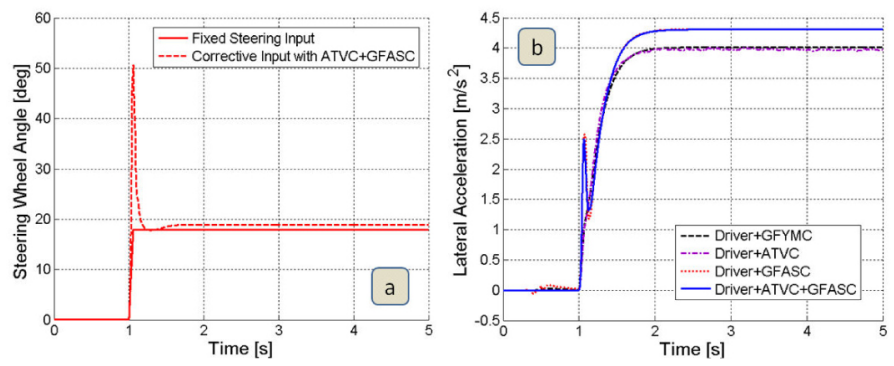

Figure 13. (a) Required steering wheel input and (b) lateral acceleration of the vehicle when driving through the step-steer maneuver using the integrated control of the ATVC and GFASC

Figure 14 illustrates the vehicle yaw rate and sideslip angle when the integrated control of the ATVC and GFASC is used, and compares the response to that obtained when the GFYMC, ATVC, and GFASC are used. As can be seen, the yaw rate and sideslip angle of the vehicle are similar to the results obtained when the GFASC is active. Table 3 summarizes the vehicle response during the step-steer test maneuver. By comparing different parameters of the vehicle response during this maneuver when it is performed with and without the integrated control of the ATVC and GFASC, we can conclude that the integrated control approach is very effective at improving all the decisive parameters of the vehicle that describe its handling characteristics.
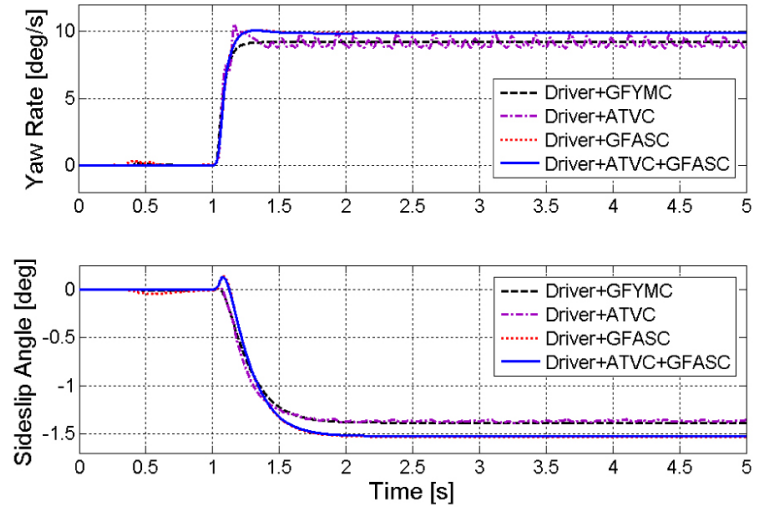

Figure 14. Vehicle yaw rate (top) and sideslip angle (bottom) when driving through the step-steer maneuver with the GFYMC, the ATVC, the GFASC, and the integrated control of the ATVC and GFASC

Table 3. Vehicle response during the step-steer maneuver using the driver model without a controller (AUTO21EV), with the GFYMC, with the ATVC, with the GFASC, and with the integrated control of the ATVC and GFASC [16]

\begin{tabular}{|c|c|c|c|c|}
\hline Parameter & $t_{\psi /}$ & $\mathrm{PO}=\frac{\dot{\psi}_{\max }-\dot{\psi}_{s s}}{\dot{\psi}_{s s}} \times 100 \%$ & $|\beta|_{\max }$ & $t_{a_{y}}$ \\
\hline AUTO21EV & $0.34 \mathrm{~s}$ & $1.30^{\circ} \%$ & $1.43^{\circ}$ & $0.66 \mathrm{~s}$ \\
\hline GFYMC & $0.15 \mathrm{~s}$ & $0.00 \%$ & $1.38^{\circ}$ & $0.51 \mathrm{~s}$ \\
\hline ATVC & $0.15 \mathrm{~s}$ & $16.1 \%$ & $1.36^{\circ}$ & $0.47 \mathrm{~s}$ \\
\hline GFASC & $0.15 \mathrm{~s}$ & $2.20 \%$ & $1.53^{\circ}$ & $0.53 \mathrm{~s}$ \\
\hline ATVC+GFASC & $0.15 \mathrm{~s}$ & $2.20 \%$ & $1.53^{\circ}$ & $0.53 \mathrm{~s}$ \\
\hline
\end{tabular}

\section{Brake-in-Turn Maneuver}

Figure 15 illustrates the vehicle trajectory relative to the desired circular path during a brake-in-turn maneuver, using the driver model with and without the integrated control of the ATVC and GFASC. As can be seen, the driver model is able to keep the vehicle on the predefined circular path while severely braking when the integrated controller is active, and the lateral deviation of the vehicle from the desired path remains negligible throughout the maneuver. Figure 16-a illustrates the driver's steering wheel input as a function of time, and indicates that the driver model [10] is able to control the vehicle very smoothly and with little steering effort when the integrated controller is used. This figure also shows the effort of the active steering controller when using the integrated control approach as it superimposes a corrective signal atop the driver's steering wheel input. Figure 16-b illustrates the lateral acceleration of the vehicle and confirms that the vehicle remains stable when the integrated 
control approach is used. This figure also confirms that the vehicle does not experience any high-frequency oscillations at high lateral accelerations, as is the case when the ATVC is used on its own. Note that, for clarity, the driver's steering input and the lateral acceleration of the vehicle when no stability controller is active are not illustrated in Figure 16.
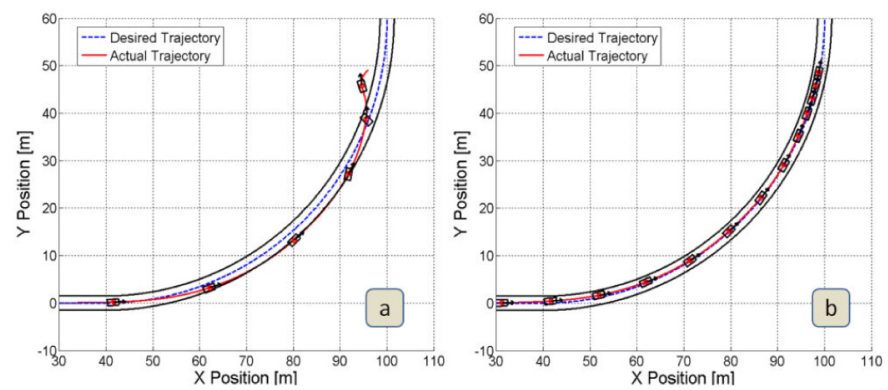

Figure 15. Desired and actual vehicle trajectories when braking in a turn using (a) the driver model only and (b) the driver model with the integrated control of the ATVC and $G F A S C$
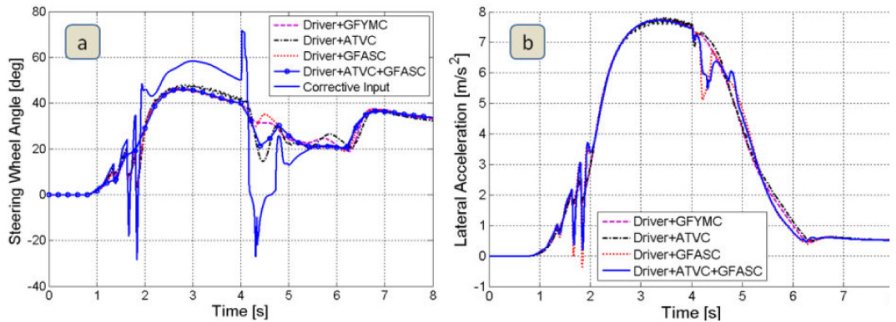

Figure 16. (a) Required steering wheel input and (b) lateral acceleration of the vehicle when braking in a turn using the driver model with the GFYMC, the ATVC, the $G F A S C$, and the integrated control of the ATVC and GFASC

Figure 17 compares the vehicle yaw rate and sideslip angle when driving through the brake-in-turn maneuver using the driver model with the GFYMC, the ATVC, the GFASC, and the integrated control of the ATVC and GFASC. Note that, for clarity, the yaw rate and sideslip angle of the vehicle when no stability controller is active are not illustrated. When the integrated control approach is used, the vehicle behaves almost like the desired reference model until the braking action begins, using primarily the GFASC because the required corrective steering angle remains below $1^{\circ}$. However, once the braking begins, both the GFASC and the ATVC contribute to minimizing the yaw rate error. As can be seen in Figure 16-a, the GFASC superimposes the driver's steering input with a steering angle of up to $2.7^{\circ}$ at 4.3 seconds of the simulation, which is equivalent to a steering wheel angle of about $48^{\circ}$, assuming a steering ratio of $18: 1$. In other words, the activation function engages the ATVC up to $90 \%$ of its full potential at 4.3 seconds in order to support the GFASC as it attempts to stabilize the vehicle.
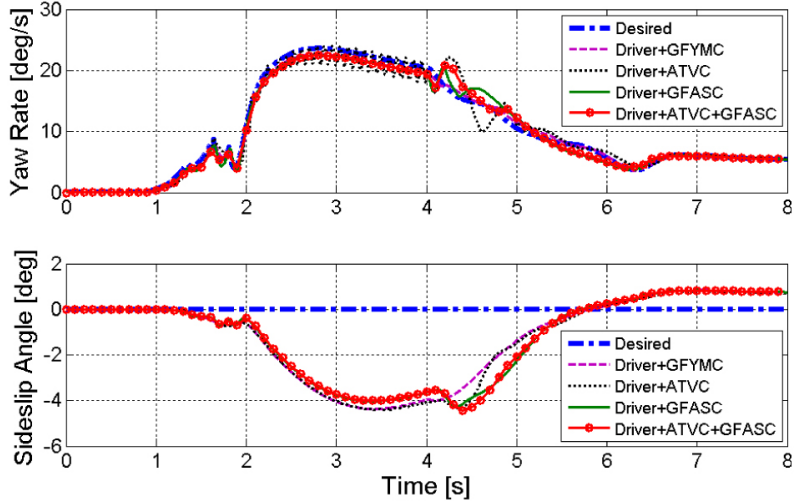

Figure 17. Desired and actual vehicle yaw rate (top) and sideslip angle (bottom) when braking in a turn using the driver model with the GFYMC, the ATVC, the GFASC, and the integrated control of the ATVC and GFASC

Figure 18 illustrates the torque of each in-wheel motor during this maneuver. Until the braking begins, the speedcontrol driver model requests up to $63 \mathrm{Nm}$ of drive torque from all four motors to keep the vehicle speed constant. When the braking begins, the ATVC increases the braking torque request on the right wheels and reduces that on the left wheels; however, at a speed of $70 \mathrm{~km} / \mathrm{h}$, the motors are not powerful enough to provide the motor torques requested by the ATVC. Braking the vehicle in a curve causes a weight shift to the front and (in this case) right side of the vehicle, which reduces the traction potential of the left tires considerably. Therefore, the slip controllers limit the braking torque of the front-left wheel between 4 and 4.1 seconds, and that of the rear-left wheel between 4.1 and 5.3 seconds, in order to avoid wheel lock-up. The slip controller for the rearright wheel limits its braking torque between 5.5 and 5.9 seconds in order to avoid a lock-up situation caused by the high available braking torque and the weight shift to the front axle. The activation of the slip controllers can be confirmed in Figure 19, which illustrates the slip ratio of each tire. Figure 20 shows the traction potential at each tire and clearly indicates that the traction potentials of the left tires have exceeded their limits. In other words, although the slip controllers have limited the braking torques on the left wheels, the high lateral acceleration of the vehicle has caused the resultant tire forces on the left wheels to exceed their traction potentials. Figure 21 illustrates the front-to-rear torque vectoring ratios and indicates that this controller also supports the driver in stabilizing the vehicle while braking in a turn, requesting more braking torque from the rear wheels than the front wheels, thereby reducing the lateral force potential at the rear axle and increasing that at the front axle. Figure 22-a illustrates the vehicle speed as a function of time and confirms the performance of the speed controller. Figure 22-b illustrates the vehicle longitudinal acceleration as a function of vehicle speed, and confirms the severity of the braking action in this maneuver. 


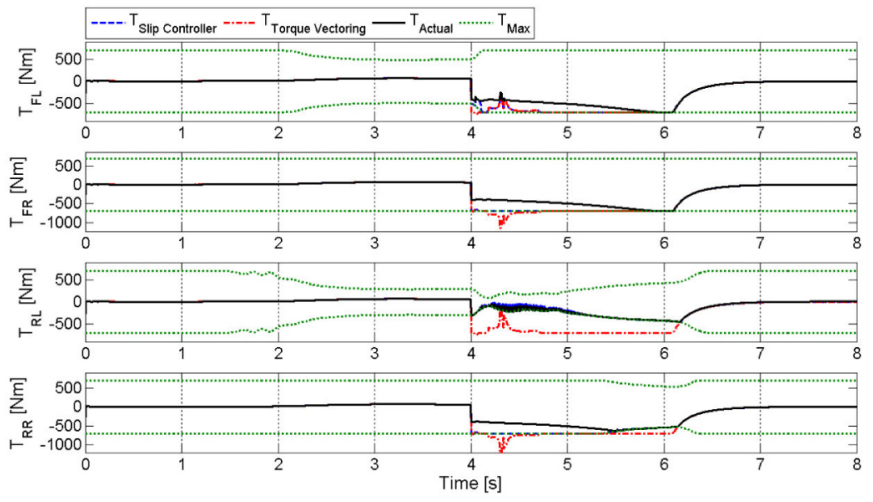

Figure 18. Requested and actual motor torques at each wheel when braking in a turn using the driver model with the integrated control of the ATVC and GFASC

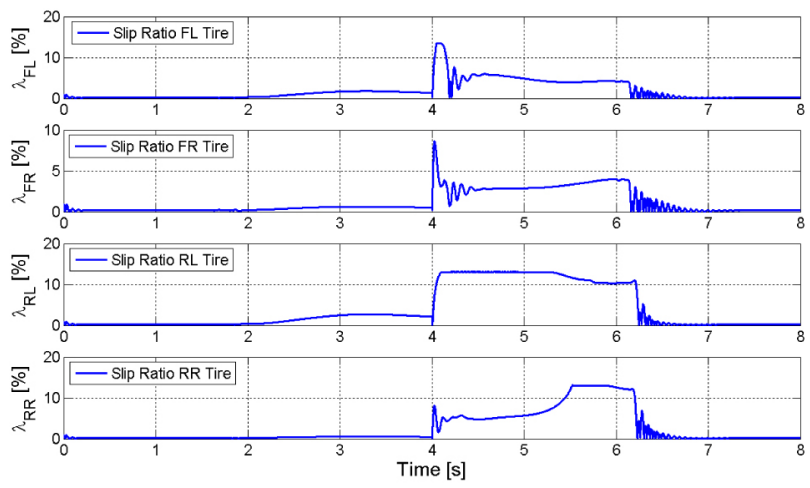

Figure 19. Tire slip ratios when braking in a turn using the driver model with the integrated control of the ATVC and GFASC
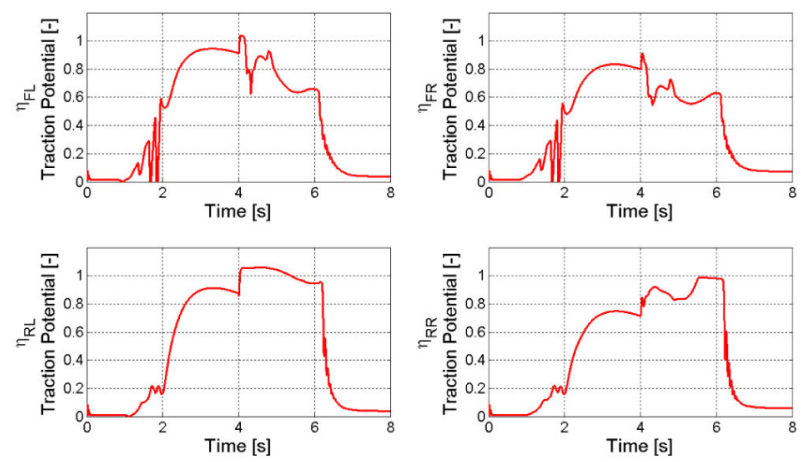

Figure 20. Traction potential of each tire when braking in a turn using the driver model with the integrated control of the ATVC and GFASC

Table 4 summarizes the vehicle response during the brake-in-turn maneuver when the integrated control approach is used, and compares it to the performance observed when no stability controller is used, and when the GFYMC, ATVC, and GFASC are used individually. Comparing different parameters of the vehicle response during this maneuver, it can be seen that the integrated control approach is very effective at improving all the decisive parameters that describe the handling, stability, and path-following capabilities of the vehicle. The integrated control of the ATVC and GFASC is able to reduce $|\beta|_{\max },|\dot{\psi}|_{\max }$, and $\left|\delta_{S W}\right|$ $\max$ substantially in comparison to the values obtained when no stability controller is used, which indicates that the integrated control approach is very effective at enhancing the vehicle handling during this maneuver.

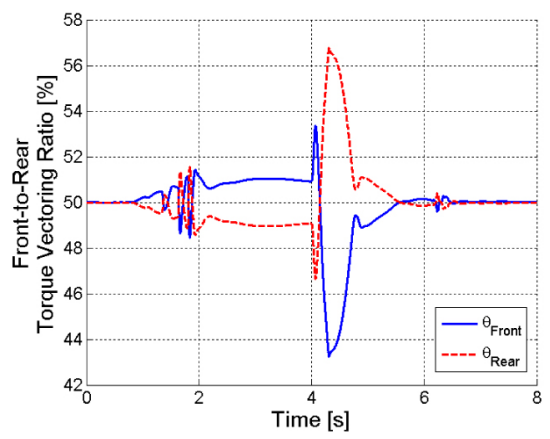

Figure 21. Front-to-rear torque vectoring ratios when braking in a turn using the driver model with the integrated control of the ATVC and GFASC
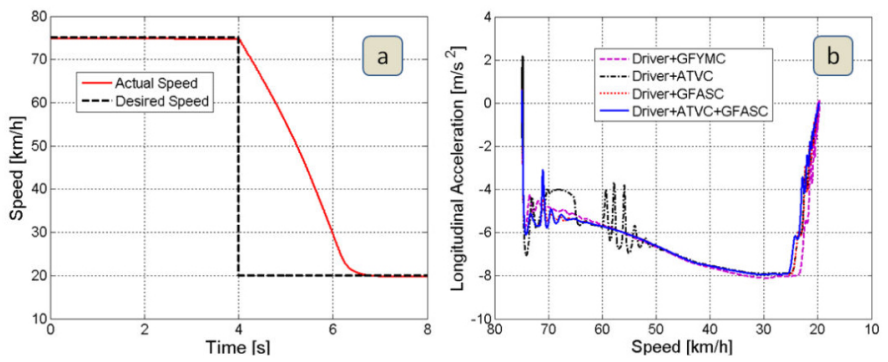

Figure 22. (a) Vehicle speed as a function of time when braking in a turn using the driver model with the integrated control of the ATVC and GFASC, and (b) longitudinal acceleration as a function of vehicle speed when braking in a turn using the driver model with the $G F Y M C$, the ATVC, the GFASC, and the integrated control of the ATVC and GFASC

Simultaneously, a reduction of $|\beta|_{\max }$ and $|\dot{\psi}|_{\max }$ indicates that the integrated control approach is also very effective at improving the vehicle stability. Finally, the fact that the maximum lateral deviation of the vehicle from the desired path remains very small throughout the maneuver confirms that the integrated control approach enhances the pathfollowing capability of the vehicle as well. 
Table 4. Vehicle response during the brake-in-turn maneuver using the driver model without a controller (AUTO21EV), with the GFYMC, with the ATVC, with the GFASC, and with the integrated control of the ATVC and GFASC [16]

\begin{tabular}{|c|c|c|c|c|}
\hline Parameter & $|\dot{\psi}|_{\max }$ & $|\beta|_{\max }$ & $\Delta y_{\max }$ & $\left|\delta_{S W}\right|_{\max }$ \\
\hline AUTO21EV & $126.5^{\circ} / \mathrm{s}$ & $50.1^{\circ}$ & $3.67 \mathrm{~m}$ & $700^{\circ}$ \\
\hline GFYMC & $22.60^{\circ} / \mathrm{s}$ & $4.36^{\circ}$ & $0.18 \mathrm{~m}$ & $46.4^{\circ}$ \\
\hline ATVC & $24.00^{\circ} / \mathrm{s}$ & $4.40^{\circ}$ & $0.18 \mathrm{~m}$ & $48.0^{\circ}$ \\
\hline GFASC & $22.45^{\circ} / \mathrm{s}$ & $4.25^{\circ}$ & $0.16 \mathrm{~m}$ & $45.7^{\circ}$ \\
\hline ATVC+GFASC & $22.45^{\circ} / \mathrm{s}$ & $4.45^{\circ}$ & $0.17 \mathrm{~m}$ & $46.1^{\circ}$ \\
\hline
\end{tabular}

\section{Straight-Line Braking on a $\mu$-Split Road}

As a final test, the AUTO21EV is driven on a $\mu$-split road and the driver model [10] attempts to stop the vehicle in an emergency braking situation. Figure 23 illustrates the vehicle trajectory for this maneuver when no stability controller is active and compares it to the case when the integrated control of the ATVC and GFASC is used. As can be seen, the integrated control approach is able to correct the side-pushing effect of the vehicle while braking on a $\mu$-split road, and prevents the vehicle from leaving the predefined road; the braking distance of the vehicle is the same as that observed when the GFASC was used on its own [13]. Looking at Figure 24, it is clear that the integrated control approach is able to limit and, later, diminish the yaw rate and sideslip angle of the vehicle while driving over the ice patch, which indicates that the vehicle remains stable during this maneuver. Note that the yaw rate and sideslip angle of the vehicle when no controller is active have been omitted from Figure 24 for clarity.

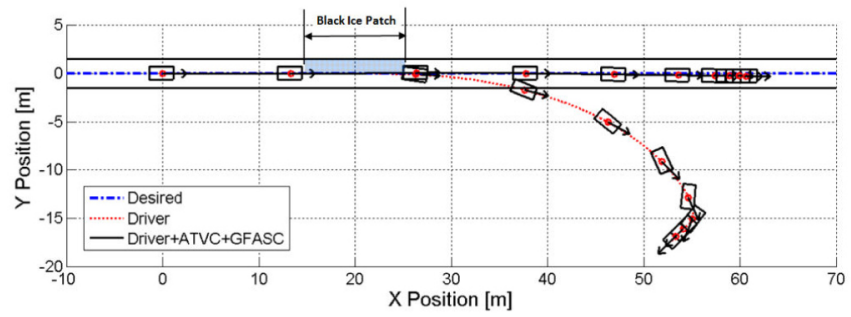

Figure 23. Desired and actual vehicle trajectories when braking on a $\mu$-split road while holding the steering wheel fixed, with and without using the integrated control of the ATVC and GFASC

Figure 25 illustrates the fixed steering wheel input and the equivalent corrective steering wheel angle generated by the GFASC to counteract the side-pushing effect of the vehicle. As can be seen, the active steering controller has applied up to $3^{\circ}$ of corrective steering angle, which is equivalent to $54^{\circ}$ of steering wheel angle, in order to correct the side-pushing effect of the vehicle. Thus, the activation function has engaged the ATVC up to $100 \%$ of its full potential in order to support the driver in stabilizing the vehicle. This level of activation is confirmed by Figure 26, which illustrates the motor torques for all four wheels. As can be seen, between 0.7 and 0.9 seconds of the simulation, the left-to-right torque vectoring controller has requested more braking torque from the left motors than the right motors in order to counteract the side-pushing effect of the vehicle. Note that the left motors are unable to generate the high braking torques requested by the ATVC due to their performance limits at high speeds, and the braking torques applied by the motors on the right side of the vehicle are adjusted accordingly. The opposite situation occurs at 1.15 seconds, when a corrective steering angle of up to $-3^{\circ}$ is generated by the GFASC. In this case, the ATVC requests more braking torque from the right motors than the left ones which are, once again, unable to meet the demands of the ATVC.
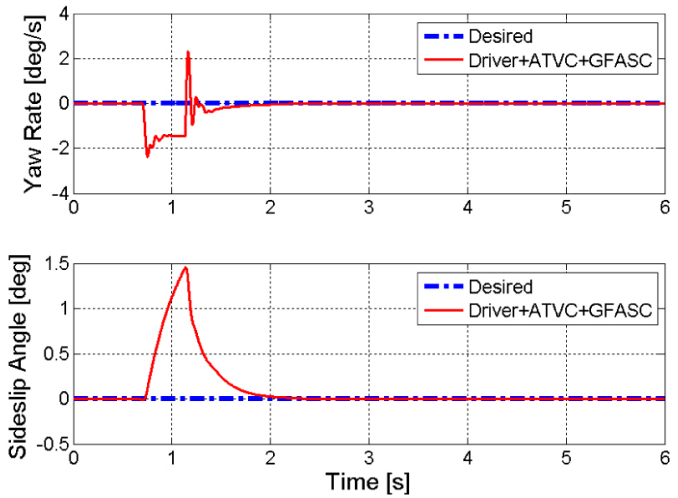

Figure 24. Desired and actual vehicle yaw rates (top) and sideslip angles (bottom) when braking on a $\mu$-split road while holding the steering wheel fixed, using the integrated control of the ATVC and GFASC

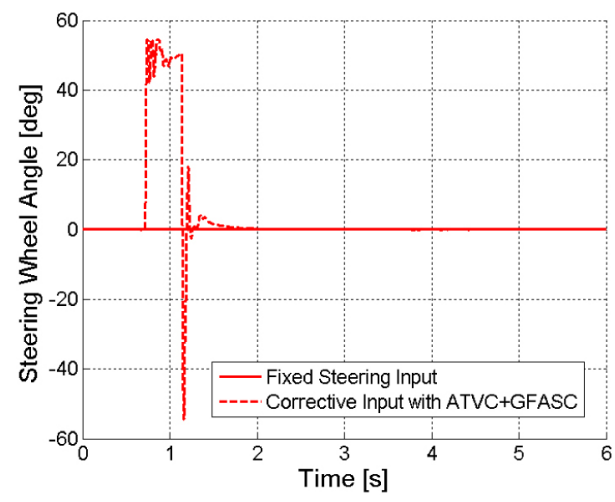

Figure 25. Equivalent corrective steering wheel input applied by the GFASC when using the integrated control of the ATVC and GFASC, augmenting the fixed steering input in order to correct the side-pushing effect of the vehicle when braking on a $\mu$-split road

Comparing Figure 26 to Figure 25 in [12], it can be confirmed that, when using the integrated control approach, the ATVC becomes activated only when the GFASC reaches 


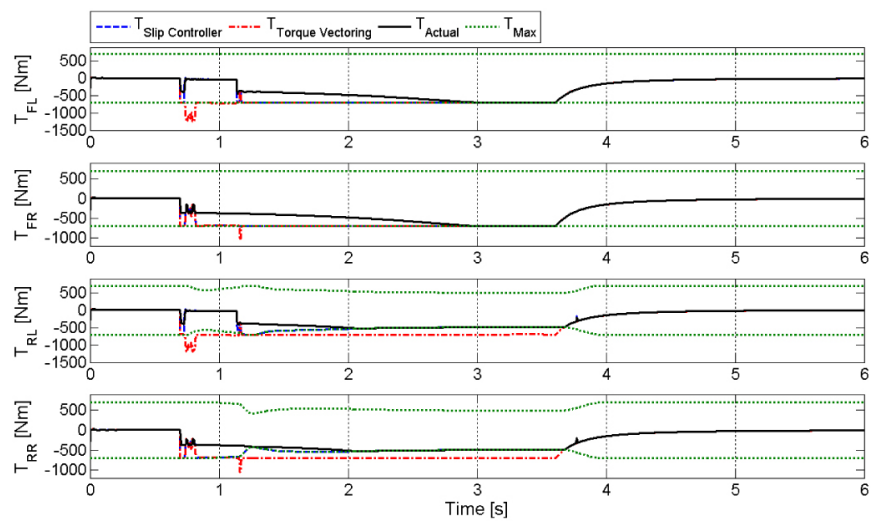

Figure 26. Requested and actual motor torques at each wheel when braking on a $\mu$-split road while holding the steering wheel fixed, using the integrated control of the ATVC and GFASC

its actuator range limits. Looking at Figure 26, it is also apparent that the slip controllers on the left wheels have limited the braking torques to $20 \mathrm{Nm}$ (between 0.7 and 1.15 seconds) in order to prevent wheel lock-up while, at the same time, ensuring the maximum possible braking force is being applied when braking on the ice patch. The activation of the slip controllers is confirmed in Figure 27, which illustrates the tire slip ratios while braking on the $\mu$-split road. Note that, later in the simulation, due to the weight shift to the front axle, the motor torques at the rear axle are limited by the maximum possible motor torques (calculated using equation (26) in [12]) to prevent wheel lock-up at higher deceleration rates. This observation is confirmed by Figure 28, which illustrates the traction potential for each tire. As can be seen, the traction potentials of the rear tires are restricted and do not exceed their limits. Figure 29 illustrates the torque distribution applied by the front-to-rear torque vectoring controller. As can be seen, this controller has requested that the front motors generate up to $52.4 \%$ of the required corrective yaw moment in order to correct the undesirable side-pushing effect when driving over the ice patch. Requesting more torque from the front motors reduces the lateral force potential on the front axle and increases that on the rear axle. The asymmetric lateral force potentials on the front and rear axles also help to generate the required corrective yaw moment around the vertical axis of the vehicle.

Table 5 summarizes the vehicle response when braking on a $\mu$-split road while holding the steering wheel fixed and using the integrated control of the ATVC and GFASC. Comparing different parameters of the vehicle response during this maneuver, it can be seen that the integrated control approach is very effective at improving all the decisive parameters that describe the stability, pathfollowing, and braking performance of the vehicle. In particular, the integrated control approach has reduced the values of $|\beta|_{\max }$ and $|\dot{\psi}|_{\max }$ substantially, which indicates an enhancement in the stability of the vehicle. The braking distance of the vehicle has also been considerably reduced by the integrated control approach, indicating an improvement in the longitudinal dynamics of the vehicle. The integrated control approach reduces the maximum lateral deviation of the vehicle as well, and prevents the vehicle from leaving the predefined road throughout the maneuver.

Table 5. Vehicle response when braking on a $\mu$-split road while holding the steering wheel fixed without a controller (AUTO21EV), with the GFYMC, with the $A T V C$, with the GFASC, and with the integrated control of the ATVC and GFASC [16]

\begin{tabular}{|c|c|c|c|c|}
\hline Parameter & $|\beta|_{\max }$ & $|\dot{\psi}|_{\max }$ & $\Delta x_{\text {braking }}$ & $\Delta y_{\max }$ \\
\hline AUTO21EV & $24.2^{\circ}$ & $63.4^{\circ} / \mathrm{s}$ & $48.4 \mathrm{~m}$ & $15.9 \mathrm{~m}$ \\
\hline GFYMC & $0.40^{\circ}$ & $1.85^{\circ} / \mathrm{s}$ & $44.5 \mathrm{~m}$ & $0.46 \mathrm{~m}$ \\
\hline ATVC & $1.70^{\circ}$ & $9.70^{\circ} / \mathrm{s}$ & $47.3 \mathrm{~m}$ & $2.70 \mathrm{~m}$ \\
\hline GFASC & $1.60^{\circ}$ & $2.50^{\circ} / \mathrm{s}$ & $45.7 \mathrm{~m}$ & $0.36 \mathrm{~m}$ \\
\hline ATVC+GFASC & $1.45^{\circ}$ & $2.35^{\circ} / \mathrm{s}$ & $45.6 \mathrm{~m}$ & $0.27 \mathrm{~m}$ \\
\hline
\end{tabular}
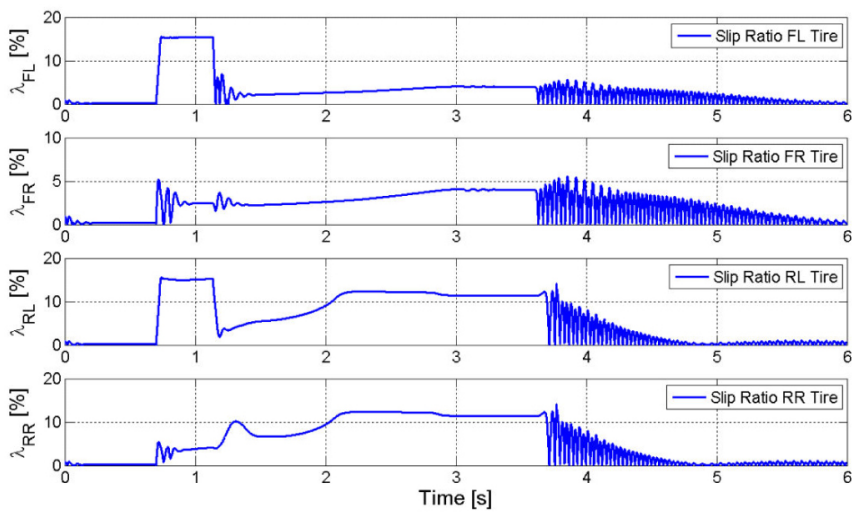

Figure 27. Tire slip ratios when braking on a $\mu$-split road while holding the steering wheel fixed, using the integrated control of the ATVC and GFASC 

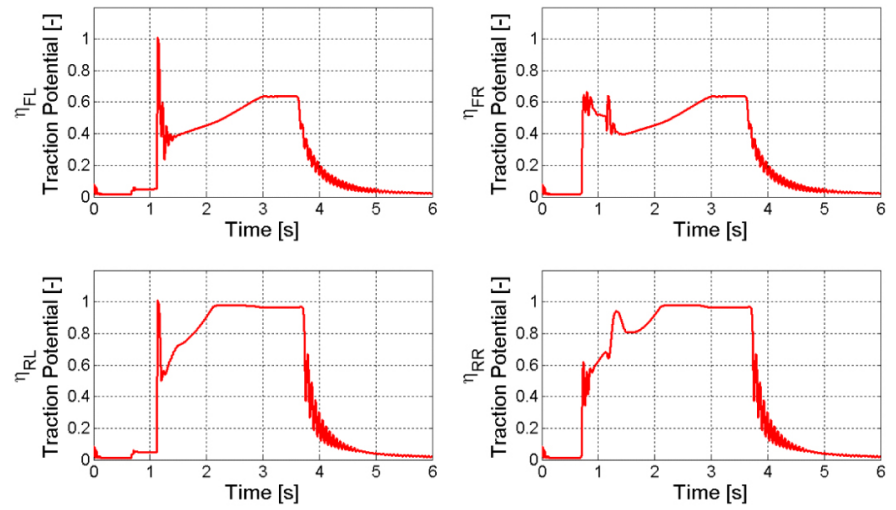

Figure 28. Traction potential of each tire when braking on a $\mu$-split road while holding the steering wheel fixed, using the integrated control of the ATVC and GFASC

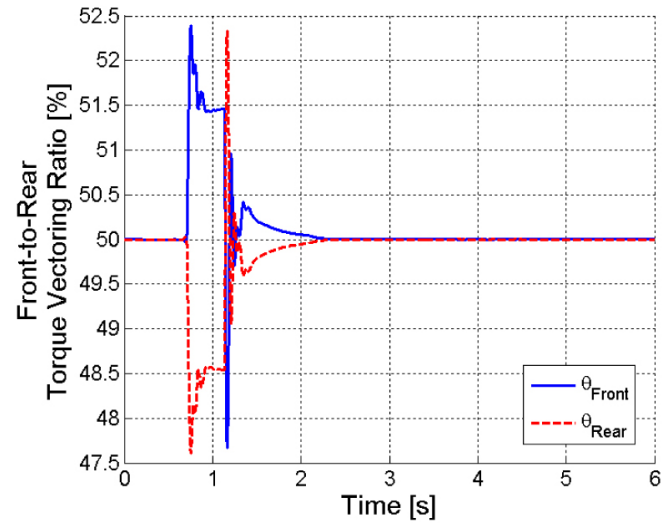

Figure 29. Front-to-rear torque vectoring ratios when braking on a $\mu$-split road while holding the steering wheel fixed, using the integrated control of the ATVC and GFASC

\section{COMPREHENSIVE EVALUATION OF CHOSEN TEST MANEUVERS}

As mentioned earlier, a comprehensive evaluation of the dynamic characteristics of a vehicle and the effectiveness of different chassis control systems can be obtained only when the results of different test maneuvers are combined and evaluated as a whole. In this section, the results of the performed test maneuvers are evaluated with respect to four main performance characteristics: handling, stability, pathfollowing capability, and longitudinal dynamics. Such an approach makes it possible to evaluate the influence of each chassis control system on the reaction of the vehicle to the steering wheel input (handling), as well as the stability, pathfollowing capability, and longitudinal dynamics of the vehicle during a maneuver. In this regard, each parameter measured during a test maneuver is assigned to one or more of the four main performance characteristics, as illustrated in Figure 30. The improvements in the dynamic behavior of the vehicle are evaluated with respect to the uncontrolled vehicle, and the effectiveness of each chassis control system is estimated using the following quantitative assessment:

$3=$ very useful, very effective

$2=$ useful, effective

$1=$ useful and effective to some extent

$0=$ no influence, ineffective

Once the effectiveness of each candidate controller has been evaluated based on the four aforementioned driving maneuvers and with respect to the four main performance characteristics, a final comprehensive evaluation is performed in which the mean value of all individual test results within a category is calculated. In this way, each candidate controller can be compared to the others in terms of the four main performance characteristics.

\begin{tabular}{|c|c|c|c|c|}
\hline Maneuver & Handling & Stability & $\begin{array}{c}\text { Path } \\
\text { following }\end{array}$ & $\begin{array}{c}\text { Longitudinal } \\
\text { dynamics }\end{array}$ \\
\hline Double-lane-change & $\begin{array}{c}|\beta|_{\max },|\dot{\psi}|_{\max },\left|\delta_{s w}\right|_{\max } \\
\Delta \dot{\psi}_{H},\left|a_{y}\right|_{\max }\end{array}$ & $|\beta|_{\max },|\dot{\psi}|_{\max }$ & & $v_{\text {bost }}$ \\
\hline Step-steer & $t_{\dot{\psi}}, t_{a_{y}}, P O,|\beta|_{\max }$ & & & \\
\hline Brake-in-turn & $|\beta|_{\max },|\dot{\psi}|_{\max },\left|\delta_{s w}\right|_{\max }$ & $|\beta|_{\max },|\dot{\psi}|_{\max }$ & $\Delta y_{\max }$ & \\
\hline $\begin{array}{c}\text { Straight-line } \\
\text { braking on a } \\
\mu \text {-split road }\end{array}$ & & $|\beta|_{\max },|\dot{\psi}|_{\max }$ & $\Delta y_{\max }$ & $\Delta x_{\text {bradurg }}$ \\
\hline
\end{tabular}

Figure 30. Important properties of the dynamic behavior of the vehicle evaluated by each test maneuver [16]

Table 6 provides a subjective evaluation of the effectiveness of the integrated control of the ATVC and GFASC based on different test maneuvers. Note that comparative tables quantifying the effectiveness of each individual control system, namely the GFYMC, ATVC, and GFASC, can be found in [16]. Figure 31 compares the performance and effectiveness of the GFYMC, ATVC, GFASC, and the integrated control of the ATVC and GFASC based on different test maneuvers. Note that the GFYMC is considered the baseline controller against which the performance and effectiveness of all other controllers are compared. As can be seen, the performance and effectiveness of the integrated control approach exceeds that of the individual control systems in all four categories. In addition, the integrated control of the ATVC and GFASC demonstrates the same performance as the GFYMC in the stability and longitudinal dynamics categories. Although the performance of the integrated control approach in the handling category cannot match that of the GFYMC, the integrated controller demonstrates better performance in the path-following category. 
Table 6. Subjective evaluation of the effectiveness of the integrated control of the ATVC and GFASC based on different test maneuvers $(3=$ very effective, 2 = effective, $1=$ effective to some extent, $0=$ ineffective)

\begin{tabular}{|c|c|c|c|c|}
\hline Maneuver & Handling & Stability & $\begin{array}{c}\text { Path } \\
\text { following }\end{array}$ & $\begin{array}{c}\text { Longitudinal } \\
\text { dynamics }\end{array}$ \\
\hline Double-lane-change & 2.5 & 3 & & 3 \\
\hline Step-steer & 3 & 3 & 3 & \\
\hline Brake-in-turn & 3 & 3 & 3 & 2.5 \\
\hline $\begin{array}{c}\text { Straight-line braking } \\
\text { on a } \mu \text {-split road }\end{array}$ & 2.83 & 3 & 3 & 2.75 \\
\hline Mean Value $\left(\bar{x}=\frac{1}{n} \sum_{i=1}^{n} x_{i}\right)$ & \multicolumn{1}{|l}{} \\
\hline
\end{tabular}

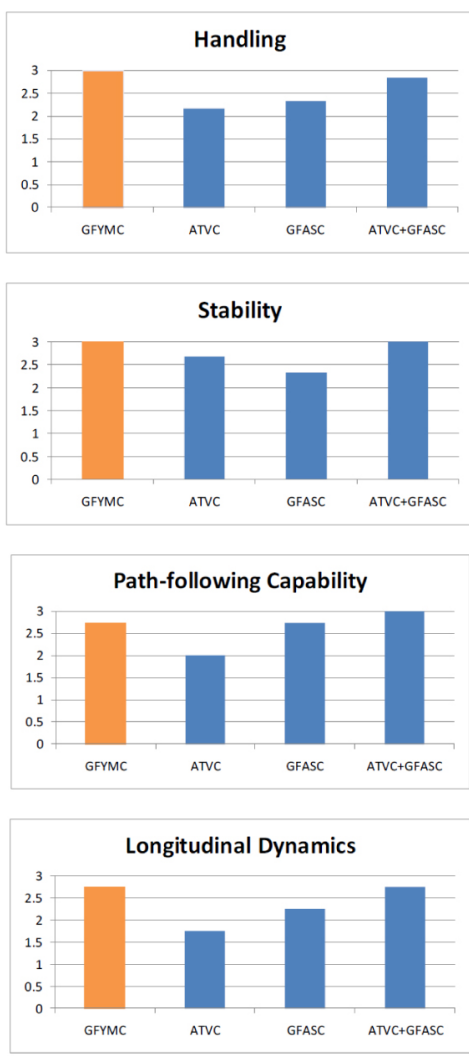

Figure 31. Subjective evaluation of the performance and effectiveness of the GFYMC, ATVC, GFASC, and integrated control of the ATVC and GFASC based on different test maneuvers $(3=$ very effective, $2=$ effective, $1=$ effective to some extent, $0=$ ineffective)

\section{EVALUATION USING A DRIVING SIMULATOR}

A hardware- and operator-in-the-loop driving simulator has been used to evaluate the performance of the integrated control system in a more realistic simulation environment. The simulator equipment used in this work is shown in Figure $\underline{32}$, and involves the synchronized operation of several timecritical computational threads. The AUTO21EV electric vehicle model has been implemented on one central processing unit (CPU) core of a quad-core real-time computer purchased from National Instruments, which uses the Laboratory Virtual Instrument Engineering Workbench (LabVIEW) Real-Time operating system to maintain precise timing during the simulation. The four in-wheel motors are simulated on the second CPU core, receiving sensor signals from the vehicle model and computing the torques applied to the wheels at each time step of the simulation. Traction controllers [9] and the advanced torque vectoring controller (ATVC) are implemented on the third CPU core. The fourth core is used for communication with a Windows-based laptop over Ethernet, and with a MotoTron electronic control module (ECM) over a Controller Area Network (CAN) bus, which is the standard in vehicular communication networks [18]. The laptop generates graphical feedback for the human driver and also receives universal serial bus (USB) interrupts generated by the steering wheel and pedals, which are relayed to the real-time computer for use as inputs to the vehicle model and controllers. The genetic fuzzy active steering controller (GFASC) has been implemented on the ECM, whose capabilities are similar to those of the controllers used in production vehicles. Real-time performance is essential to maintain the illusion of reality for both the human driver (the operator in the simulation loop) and the ECM (the hardware in the simulation loop). The integrated control system is evaluated using three of the maneuvers used above: the ISO double-lane-change maneuver, the brake-in-turn maneuver, and straight-line braking on a $\mu$-split road.

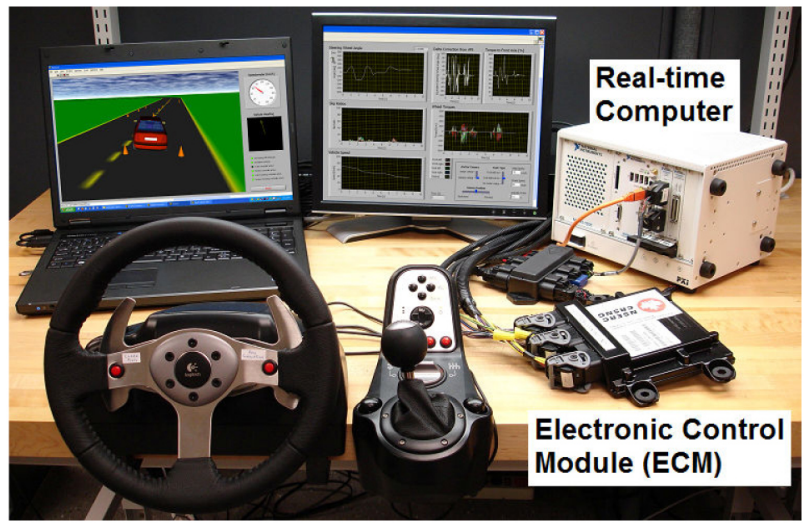

Figure 32. Hardware- and operator-in-the-loop driving simulator equipment, consisting of input devices (steering wheel and pedals), a Windows-based laptop computer, a quad-core real-time computer, and an electronic control module (ECM) that communicates with the real-time computer over a CAN bus

\section{ISO Double-Lane-Change Maneuver}

We first consider the ISO 3888 double-lane-change maneuver described earlier; however, the initial speed is reduced to $60 \mathrm{~km} / \mathrm{h}$ to make this maneuver feasible for a nonprofessional human driver. Even with this speed reduction, 
the maneuver was found to be challenging without the assistance of the controllers. The maneuver became easier to complete successfully when aided by either the ATVC or the GFASC, but was easiest when the integrated controller was used. Shown in Figure 33-a are the vehicle trajectories when no controllers were active and when the integrated controller was active (the maneuver was also successfully completed when using either the ATVC or the GFASC); however, as shown in Figure 33-b, the least amount of driver effort was required when using the integrated control of the ATVC and GFASC. Note, in particular, the reduction in the steering wheel angle required when entering the left lane (between 0.5 and 1.5 seconds of the simulation) and when completing the second lane change (between 3 and 4 seconds). Figure 34-a shows the same performance metric presented in Figure 8-d, again indicating that the steering performance of the vehicle is best when using the integrated control strategy. The effects of the ATVC are evident in Figures 34-b and 34-c, where the phases of the maneuver are echoed in the distribution of torques among the four in-wheel motors. Finally, Figure 34-d illustrates the effect of the GFASC, where the steering wheel input provided by the driver is compared to the equivalent steering wheel angle upon adding the correction specified by the GFASC. Note that the corrected steering wheel angle leads the input from the driver, moving it closer to the steering wheel angle required when no controllers were active (Figure 33-b, No Controllers curve).
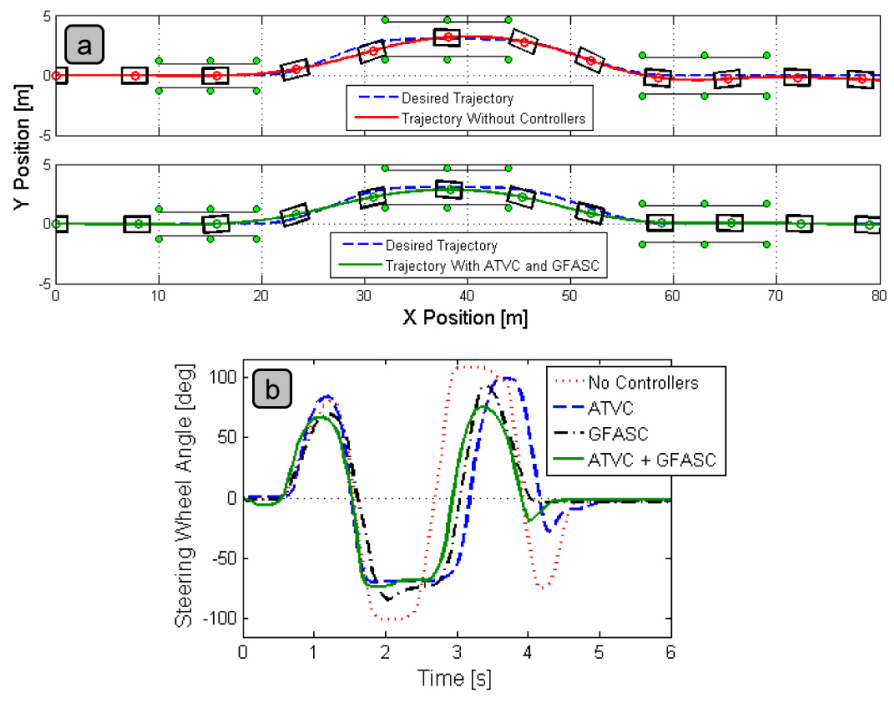

Figure 33. Vehicle trajectory without the assistance of any controllers and with the integrated control of the ATVC and GFASC (a), and driver's steering wheel input without controllers, with the ATVC, with the GFASC, and with the integrated controller (b) during the doublelane-change maneuver performed on the driving simulator
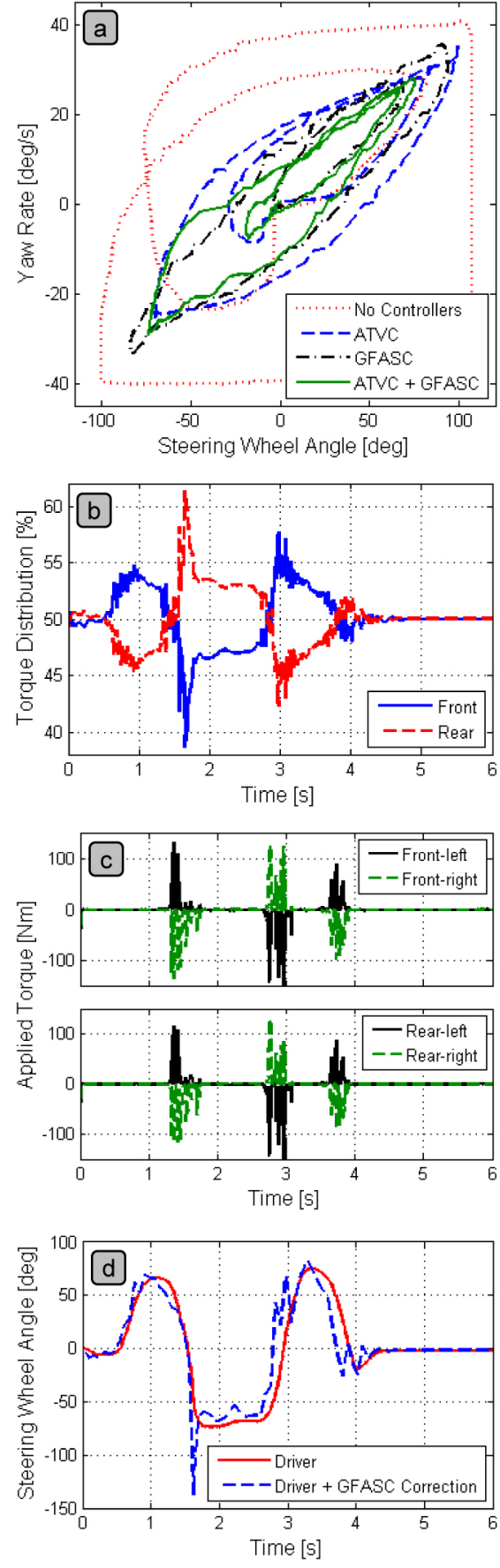

Figure 34. Vehicle yaw rate as a function of steering wheel input (a), front-to-rear torque distribution (b), wheel torques (c), and equivalent steering wheel angle upon applying the correction from the GFASC (d) during the double-lane-change-maneuver performed on the driving simulator, using the integrated control of the $A T V C$ and GFASC

\section{Brake-in-Turn Maneuver}

We now consider the brake-in-turn maneuver. As before, the vehicle enters a curve of radius $60 \mathrm{~m}$ while traveling at a 

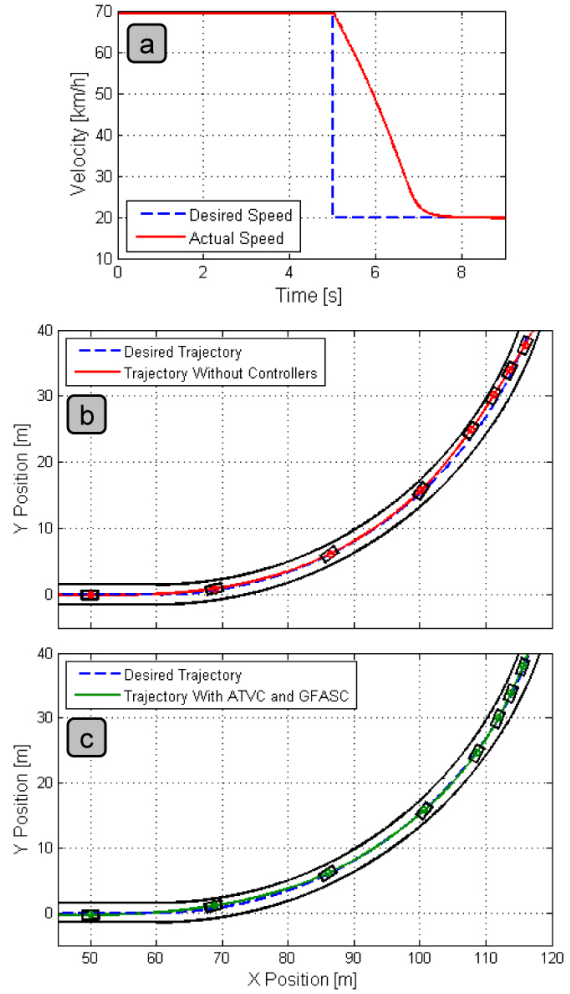

Figure 35. Vehicle speed (a), trajectory without the assistance of any controllers (b), and trajectory with the integrated control of the ATVC and GFASC (c) during the brake-in-turn maneuver performed on the driving simulator

constant speed. To make this maneuver feasible for a nonprofessional human driver, the initial speed is set to $70 \mathrm{~km} / \mathrm{h}$. Once a steady-state yaw rate has been reached, the brakes are applied until the speed has been reduced to $20 \mathrm{~km} / \mathrm{h}$ (Figure 35-a).

Shown in Figures 35-b and 35-c are, respectively, the vehicle trajectories when no controllers were active and when using the integrated controller (the maneuver was also successfully completed when using only one of the two controllers). As suggested by the steering wheel input provided by the driver (Figure 36-a), the maneuver was most challenging when no controllers were active, somewhat less challenging when assisted by either the ATVC or the GFASC, and least challenging when using the integrated controller. Note, in particular, the smooth steering input and the lack of counter-steering required when assisted by both controllers, which also results in a smoother yaw rate (Figure 36-b) and, thus, a more comfortable ride. The front-to-rear torque distribution ratio and the torques applied to each wheel are shown in Figures 36-c and 36-d, respectively. Note that the ATVC provides assistance in normal driving conditions (before 5 seconds, while turning at a constant speed) as well as more extreme situations (after 5 seconds, once the brakes have been applied). Also note that the slip controllers have limited the torques on the rear-left and rear-right wheels (from 5.1 seconds and 6.3 seconds, respectively) to prevent tire spin-out. Finally, the effect of the GFASC is illustrated in Figure 36-e, where the steering wheel angle provided by the driver is compared to the equivalent steering wheel angle once the correction specified by the GFASC has been added. The GFASC has provided the counter-steering necessary to keep the vehicle on the desired path and has reacted faster than would a human driver, thereby enhancing the overall stability and maneuverability of the vehicle.

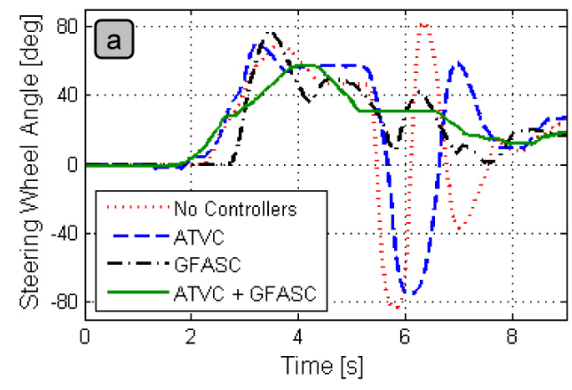

Figure 36. Driver's steering wheel input (a), vehicle yaw rate (b), front-to-rear torque distribution ratio (c), applied wheel torques (d), and equivalent steering wheel angle upon applying the correction from the GFASC (e) during the brake-in-turn maneuver performed on the driving simulator, using the integrated control of the $A T V C$ and GFASC 

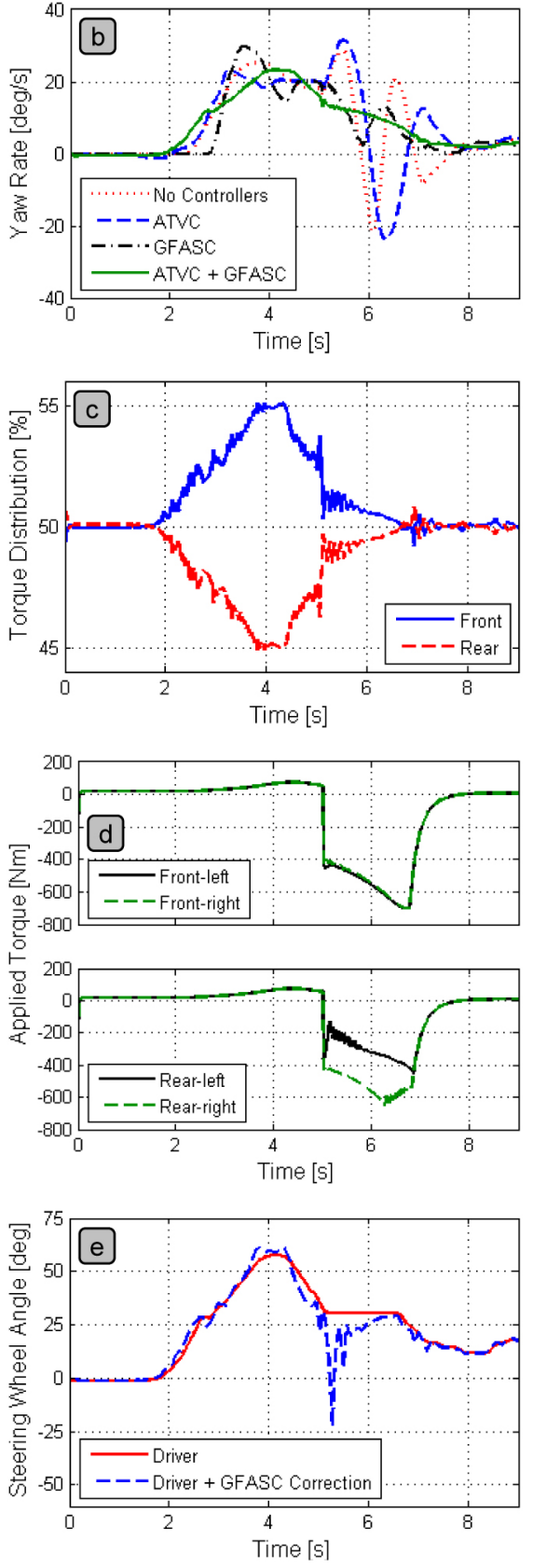

Figure 36. (cont.) Driver's steering wheel input (a), vehicle yaw rate (b), front-to-rear torque distribution ratio (c), applied wheel torques (d), and equivalent steering wheel angle upon applying the correction from the GFASC (e) during the brake-in-turn maneuver performed on the driving simulator, using the integrated control of the ATVC and GFASC

\section{Straight-Line Braking on a $\mu$-Split Road}

The last maneuver we consider is braking on a $\mu$-split road, again with the driver holding the steering wheel fixed at zero. As shown in Figure 37-a, the vehicle is aggressively braked from a speed of $80 \mathrm{~km} / \mathrm{h}$ to $5 \mathrm{~km} / \mathrm{h}$. Shown in Figure $\underline{37-b}$ are the trajectories of the vehicle when unassisted by any controllers, when assisted by either the ATVC or the GFASC, and when assisted by the integrated control of the ATVC and GFASC. Clearly, the best trajectory is obtained when the GFASC controller is active, regardless of whether the ATVC is also enabled. However, the severity of the correction applied by the GFASC is reduced substantially when the ATVC is also enabled (Figure 38-c). The front-torear torque distribution ratio and the torques applied to each wheel are shown in Figures 38-a and 38-b, respectively. Note that the traction controllers have prevented lock-up on the left wheels when driving over the ice patch (between 0.7 and 1.2 seconds of the simulation).
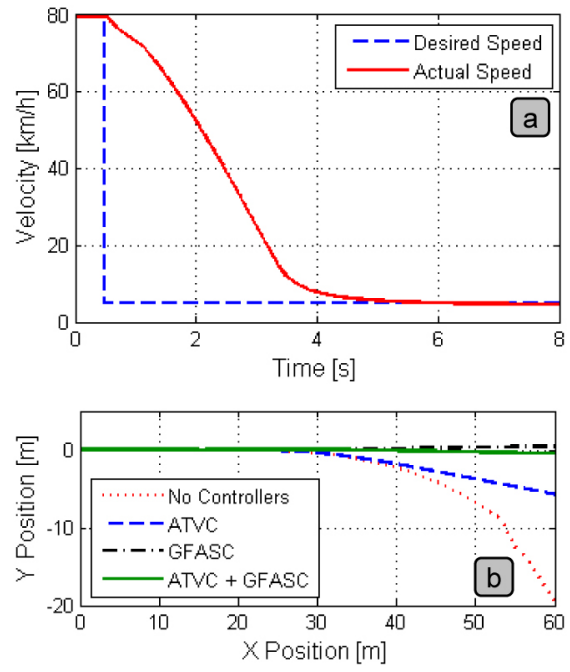

Figure 37. Target and actual vehicle speeds (a), and vehicle trajectory without the assistance of any controllers and with the integrated controller (b) when braking on a $\mu$-split road using the driving simulator

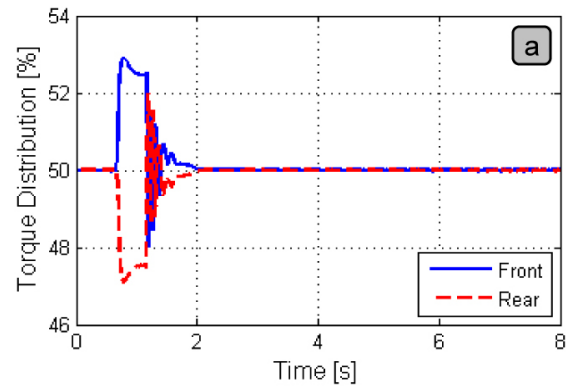

Figure 38. Front-to-rear torque distribution ratio (a), applied wheel torques (b), and equivalent corrective steering wheel angle applied by the GFASC (c) when braking on a $\mu$-split road using the driving simulator, assisted by the integrated control of the ATVC and GFASC 

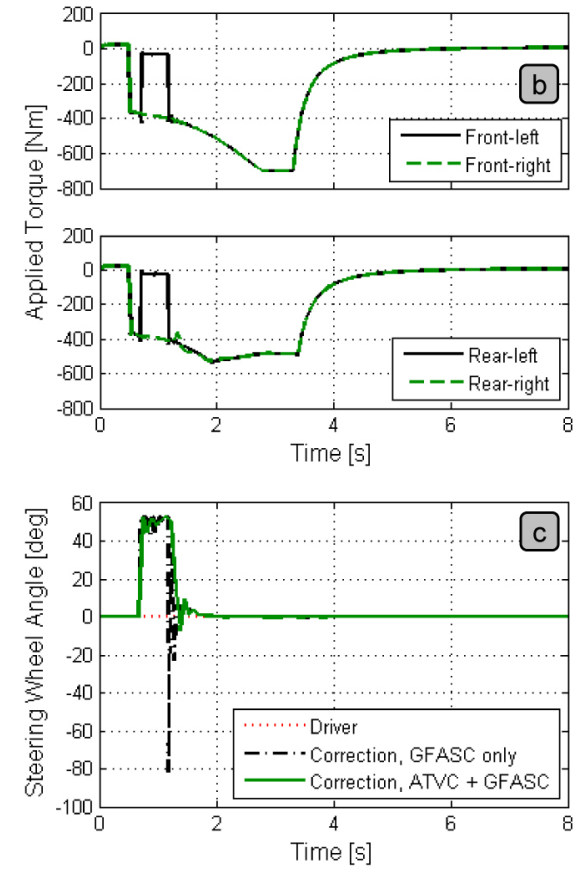

Figure 38. (cont.) Front-to-rear torque distribution ratio (a), applied wheel torques (b), and equivalent corrective steering wheel angle applied by the GFASC (c) when braking on a $\mu$-split road using the driving simulator, assisted by the integrated control of the ATVC and GFASC

\section{CONCLUSIONS}

In this work, the integration of an advanced torque vectoring controller and a genetic fuzzy active steering controller is studied. In order to investigate the capabilities of such a system, an electric vehicle model has been developed with four direct-drive in-wheel motors and an active steering system. Using this vehicle model, an advanced slip control system, an advanced torque vectoring controller, and a genetic fuzzy active steering controller were developed in the past. Comparing the performance and effectiveness of the individual control systems from previous work, it has been found that the intervention of the genetic fuzzy active steering controller is considered to be a continuous process, and is not perceived by the driver as being disruptive or annoying. It is also advantageous to employ steering intervention rather than braking or driving individual wheels when controlling the vehicle on slippery surfaces, since steering intervention requires less frictional force between the tire and the road to generate a corrective yaw moment. However, the genetic fuzzy active steering controller suffers from its limited range of effectiveness (caused by actuator restrictions). The advanced torque vectoring controller, on the other hand, is found to be very effective at improving the vehicle stability and handling, even when the vehicle is driven near its handling limits. However, it has also been observed that the actuation of the in-wheel motors to generate a corrective yaw moment can cause some oscillations in the vehicle states, which might be perceived by the driver as being disruptive. To overcome the shortcomings of each of these control systems, a novel activation function is introduced in this work that takes advantage of the strengths of each chassis control system and distributes the required control effort between the in-wheel motors and the active steering system based on the difference between the desired and actual behavior of the vehicle. The performance and effectiveness of the integrated approach are evaluated using several test maneuvers in the simulation environment. It is confirmed that the integrated control approach performs better than each individual control system in all test maneuvers. Finally, the performance of the integrated control strategy has been demonstrated on a driving simulator, and its effectiveness compared to each individual control system has been confirmed.

\section{ACKNOWLEDGMENTS}

Funding for this work was provided by the Natural Sciences and Engineering Research Council of Canada and a grant from AUTO21, a Canadian Network of Centres of Excellence.

\section{REFERENCES}

1. Federal Motor Vehicle Safety Standard (FMVSS) No. 126: Electronic Stability Control Systems, Docket No. NHTSA-2007-27662, 2007.

2. ATZ magazine: "Automobiltechnische Zeitschrift", ISSN 0001-2785-10810, May, 2006.

3. Koehn, P., Eckrich, M., Smakman, H., and Schaffert, A., "Integrated Chassis Management: Introduction into BMW's Approach to ICM," SAE Technical Paper 2006-01-1219, 2006, doi:10.4271/2006-01-1219.

4. Semmler, S., Rieth, P., and Linkenbach, S., "Global Chassis Control The Networked Chassis," SAE Technical Paper 2006-01-1954, 2006, doi: $10.4271 / 2006-01-1954$.

5. Gordon, T., Howell, M., and Brandao, F.: "Integrated control methodologies for road vehicles", Vehicle System Dynamics, 40(1-3), pp. 157-190, 2003.

6. Yamamoto, M., "Active Control Strategy for Improved Handling and Stability," SAE Technical Paper 911902, 1991, doi: 10.4271/911902.

7. Andreasson, J., Knobel, C., and Bünte, T.: "On road vehicle motion control - Striving towards synergy", Proceedings of the 8th International Symposium on Advanced Vehicle Control, Taipei, AVEC060209, 20-24 August 2006.

8. Smart Canada, fortwo coupe specifications, 2010. http:// www.thesmart.ca

9. Jalali, K., Uchida, T., McPhee, J., and Lambert, S., "Development of a Fuzzy Slip Control System for Electric Vehicles with In-wheel Motors," SAE Int. J. Alt. Power. 1(1):46-64, 2012, doi:10.4271/2012-01-0248.

10. Jalali, K., Lambert, S., and McPhee, J., "Development of a Pathfollowing and a Speed Control Driver Model for an Electric Vehicle," SAE Int. J. Passeng. Cars - Electron. Electr. Syst. 5(1):100-113, 2012, doi: $10.4271 / 2012-01-0250$

11. Jalali, K., Uchida, T., McPhee, J., and Lambert, S., "Integrated Stability Control System for Electric Vehicles with In-wheel Motors using Soft Computing Techniques," SAE Int. J. Passeng. Cars - Electron. Electr. Syst. 2(1):109-119, 2009, doi:10.4271/2009-01-0435.

12. Jalali, K., Uchida, T., Lambert, S., and McPhee, J., "Development of an Advanced Torque Vectoring Control System for an Electric Vehicle with In-Wheel Motors using Soft Computing Techniques," SAE Int. J. Alt. Power. 2(2): in press, 2013, doi:10.4271/2013-01-0698.

13. Jalali, K., Uchida, T., McPhee, J., and Lambert, S., "Development of an Advanced Fuzzy Active Steering Controller and a Novel Method to Tune the Fuzzy Controller," SAE Int. J. Passeng. Cars - Electron. Electr. Syst. 6(1):241-254, 2013, doi:10.4271/2013-01-0688.

14. Roenitz, R., Braess, H.H., and Zomotor, A.: "Methods and criteria for evaluation of the behavior of the passenger vehicle - Part I" " "Verfahren und Kriterien zur Bewertung des Fahrverhaltens von Personenkraftwagen - Stand und Problematik, Teil 1"), ATZ 
Automobiltechnische Zeitschrift, $1 / 77$ and 3/77, pp. 29-38 and 39-46, 1977.

15. Roenitz, R., Braess, H.H., and Zomotor, A. "Methods and criteria for evaluation of the behavior of the passenger vehicle - Part II" ("Verfahren und Kriterien zur Bewertung des Fahrverhaltens von Personenkraftwagen - Ein Rueckblick auf die letzten 20 Jahre, Teil 2"), ATZ Automobiltechnische Zeitschrift, 99/100, pp. 780-786, 1998.

16. Jalali, K.: Stability Control of Electric Vehicles with In-wheel Motors: A New Approach Using Soft Computing Techniques, LAP LAMBERT Academic Publishing, 2012.

17. Bauer, H.: Driving Safety Systems, 2nd edition, Robert Bosch GmbH, Warrendale, 1999.

18. Bosch Automotive Handbook, 6th edition, Robert Bosch GmbH, Plochingen, 2004. 